\title{
TENDIENDO PUENTES ENTRE LA TEORÍA Y LA PRÁCTICA CIENTIFÍCA: EL PÉNDULO BALÍSTICO (Robins, 1742; Hutton, 1775)
}

Encarna HIDALGO CAMARA

Dpto De Lógica y Filosofía de la Ciencia. UNED

\section{1.- La "esquizofrenia" de la artilleria}

En el siglo XVIII, en materia de artillería, coexistieron la teoría parabólica arropada por la práctica tradicional (tendencia predominante dentro del colectivo de los artilleros), junto con una intensa labor de investigación en todas las ramas de la matemática y muy notablemente en la matemática mixta, en la que se encuadra la mecánica racional ${ }^{1}$. El problema balístico entró en el terreno de ésta última desde el momento en que se admitió que la resistencia del aire intervenía en la determinación de la trayectoria de los proyectiles, y se intentó su resolución por medio del análisis, fundamentalmente a partir de la ley de resistencia cuadrática expuesta por Newton en el Libro II de sus Principia ${ }^{2}$.

${ }^{1}$ Testigos de esta efervescencia investigadora son las publicaciones periódicas de las sociedades cientificas, en las que ha quedado constancia de los múltiples problemas matemáticos y mecánicos a los que se buscaba respuesta, y de como éstos evolucionaban desde los supuestos más sencillos a formulaciones más elaboradas. Estas publicaciones son imprescindibles a la hora de comprender el desarrollo de la artillería, pues el problema balístico fue continuamente reformulado en ellas, ampliándose cada vez más el número de factores que en él se reconocían. Son especialmente relevantes los distintos estudios publicados en esta época en Histoire de la Académie Royale de Sciences de Paris, Philosophical Transactions, Acta Eruditorum, Commentarii Academiae Scientiarum Imperialis Petropolitanae, Histoire de l'Académie Royale des Sciences et Belles Lettres de Berlin y el Joumal de l'École Polytechnique.

2 La resistencia que ejerce el aire sobre un proyectil está en razón compuesta del cuadrado de la velocidad de éste, del cuadrado de su diámetro y de la densidad del aire. El espacio recorrido por el proyectil se representa gráficamente por una curva aproximadamente hiperbólica. NEWTON, Isaac; Principia Mathematica Philosophia Naturalis, $1^{3}$ ed. en 1687. Trad. española de Eloy Rada en Alianza Editorial, Madrid (1987), 2 vols. "Libro II. Del movimiento de los cuerpos", pp. 413609 , vol. $2^{2}$.

Éndoxa: Series Filosóficas, $n^{\circ} 2,1993$, UNED, Madrid:

Encarne Hidalgo: Tendiendo puentes entre la teoría y la práctica cientifica: el péndulo balístico. pp. 237-281 
Si repasamos las obras claves de la historia de la artillería, centrándonos en las aparecidas en el siglo XVIII, distinguiremos en ellas cuatro tipos de fuentes: 1) los habituales tratados de artilleria, que seguian un mismo orden expositivo (teoria de pólvoras, clasificación de piezas, descripción de éstas, modos de hacer puntería, uso de algunos instrumentos sencillos, minas etc.). Su contenido se mantiene en gran parte intacto durante casi todo el siglo XVIII y sus autores generalmente son militares. Estos tratados pueden llegar a imprimirse o quedarse en un conjunto de cuadernos dictados en las clases. En España son ejemplos de lo primero los libros de Fernández de Medrano ${ }^{3}$, Rovira ${ }^{4}$, Díaz Infante ${ }^{5}$, Morla ${ }^{6}$ etc.

2) Cursos o compendios de matemáticas en los que se dedica algún capitulo a la artillería; la calidad de estos capítulos depende de la calidad del curso y sus autores no suelen ser militares, aunque puedan estar relacionados con la docencia en las academias castrenses, por ejemplo, Tosca ${ }^{7}$ o Bézout ${ }^{\dot{8}}$.

3 FERNANDEZ DE MEDRANO, Sebastián; El perfecto artificial, bombardero y artillero, gue contiene los artificios de fuegos marciales, nueoo uso de bombas, granadas, y práctica de la artilleria y mosquete etc. Bruselas (1699).

4 ROVIRA, Francisco Javier; Tratado de artillería para el uso de los caballeros. Guardias-Marinas en su Academia. Por D. Franciso Xavier Rovira, Teniente de navio de la Real Armada, y profesor de Artillería en la misma Academia. Dedicado a la inmortal memoria del Excmo. Señor Don Jorge Juan. En la Imprenta de la misma Academia (1773).

5 DIAZ INFANTE, José; Compendio de artillería para el servicio de la Marina, (1754).

6 MORLA, Tomás de; Tratado de artillería, para el uso de la Academia de Caballeros Cadetes del Real Cuerpo de Artilleria. 4 vols., $2^{\mathbf{2}}$ ed. Segovia (1816) (1 $1^{\mathrm{a}}$ ed. 1784-1786).

7 TOSCA, Tomás Vicente; "Tratado XVII. De la pirotecnia, arte tormentaria o Artillería" en Compendio Mathematico en que se contienen todas las materias más principales de las ciencias que tratan la cantidad. Tomo V. Segunda impresión, Madrid (1727). Primera ed. Valencia (1707-1715). Tosca nop estuvo vinculado a ninguna institución militar, lo que no impidió que el contenido de su tratado fuese profusamente utilizado en la enseñanza militar española de gran parte del XVIII.

8 BÉZOUT; Cours de mathématiques á l'usage du corps royal de l'artillerie. Paris (1770-1772). 
3) Los trabajos matemáticos que aparecen en las publicaciones científicas. Sin duda constituyen la investigación puramente balística, es decir físico-matemática, más arriesgada. Durante la práctica totalidad del siglo sus autores son matemáticos que nunca tuvieron nada que ver con el ejército, es el caso de Euler ${ }^{9}$, los Bernoulli $^{10}$, Lambert ${ }^{11}$ etc.

4) Libros que no se ajustan al planteamiento tradicional de los tratados de artillería y que vienen a ser los primeros tratados de balística propiamente dichos. Les diferencia de los trabajos aparecidos en las revistas su extensión y su acercamiento a la vía experimental. Valgan como ejemplo las obras de Bélidor ${ }^{12}$ y de Robins ${ }^{13}$.

Fácilmente se observará que estos cuatro tipos de fuentes son obra de dos colectivos bien diferenciados: los artilleros y los matemáticos. La historia de la artillería en el XVIII es la historia de los trabajos de estas dos comunidades, que frecuentemente, y en especial en la primera mitad del siglo, coexisten independiente-

9 EULER; "Recherches sur la véritable courbe que décrivent les corps jettés dans l'air ou dans un autre fluide quelconqe", Histoire de l'académie Royales des Sciences et Belles Lettres de Berlin (1753) pp. 321-352. Berlin (1755).

10 Por ejemplo, BERNOULLI, Johann; "Responsio ad nonneminis provocationem, ejusque solutio quaestionis ipsi ab eodem propositae de invenienda Linea curva quam describis projectile in medio resistente" en Acta Eruditorum, Leipzig, Mayo 1719, pp. 217-226. BERNOULLI, Daniel, J. F.; "Theorema de motu curvilineo corporum, quae resistentiam patiuntur velocitatis suae quadrato proportionalem una cum solutione problematis in Act. Lips. M. Nov. 1728 propositi." en Commentarii Academiae Scientiarum Imperialis Petropolitanae, tomo IV, pp. 136-143 (1729).

11 LAMBERT; "Memoire sur la resistance des fluides avec la solution du probleme balistique" en Histoire de l'Academie Royale de Berlin (1767), pp. 102-188.

12 BELIDOR; Le bombardier françois, ou nouvelle methode de jetter les bombes aoec precision .Par M. Belidor, comissaire ordinaire de l'Artillerie, professeur royal des Mathematiques aux Ecoles du même Corps, Membre des Academies Royales des Sciences $d^{\prime}$ Angleterre et de Prusse, correspondant de celle de Paris. Paris (1731).

13 ROBINS, Benjamin; New principles of Gunnery (1742); traducción francesa de la traducción alemana comentada por Leonard Euler: Nouveaux principes d'artillerie, de M. Benjamin Robins, commentés par M. Leonard Euler, traduit de l'allemand avec des notes, par M. Lombard, professeur royal aux Écoles d'Artillerie à Auxonne. Dijon (1783). 
mente. Entender el tipo de relación que se estableció entre ambos colectivos es la clave del desarrollo de la balística.

Por parte militar, en el siglo XVIII se produce la institucionalización definitiva y generalizada de la artillería en academias o escuelas dependientes de los respectivos ejércitos, con las variantes propias de cada país. En estas academias se recoge la enseñanza artillera tradicional, centrada en la teoría parabólica, con un gran peso de la práctica artillera - que es, en definitiva, la que marca el grado de aceptación de una teoría balística dentro del ejército - y todavía con una importante dosis de escepticismo acerca de la importancia del estudio de la matemática para un artillero. Esto último precisa una urgente matización: a mediados de siglo en los círculos ilustrados no se discute la necesidad de implantar el estudio de las matemáticas en los centros militares, y sólo en España se contabiliza ya cerca de una docena de instituciones de este tipo que se rigen por este criterio. Otra cosa bien distinta es el contenido de esta enseñanza, y en particular la introducción del cálculo diferencial e integral, así como el grado de inclinación que el colectivo de los artilleros muestre hacia el estudio de las "matemáticas sublimes"14.

A pesar de que en los resultados obtenidos en la práctica artillera habitual se apreciaban divergencias notables respecto a los predichos por las teorías matemáticas, especialmente por la newtoniana, estos resultados siguieron constituyendo durante gran parte del XVIII la razón última en la que los artilleros basaban su fidelidad a la teoría parabólica. No obstante, es necesario advertir que en el caso de la artillería difícilmente puede esgrimirse la experiencia como el argumento definitivo que consagre una teoría, cualquiera que ésta sea, pues la falta de uniformización en todo el material, piezas, pólvoras, proyectiles e instrumentos, era tal que incluso una misma experiencia repetida en distintas ocasiones

14 En la época, se denominaban "matemáticas sublimes" al cálculo diferencial e integral. 
podía arrojar resultados contradictorios ${ }^{15}$. Por ejemplo, con motivo de una memoria aparecida en las Philosophical Transactions en la que, tras repetidas experiencias, se dice haber observado que los proyectiles disparados con un arma de fuego y una carga determinada, sufren un desplazamiento lateral de derecha a izquierda, la Academia de Ciencias de París propuso estudiar este fenómeno repitiendo las mismas experiencias y siguiendo paso a paso las indicaciones. El encargado de realizar la comprobación fue Cassini hijo, y los resultados obtenidos fueron completamente contrarios a los expuestos ${ }^{16}$.

Por su parte, en el terreno de la filosofía natural reinaba la razón matemática ${ }^{17}$. En aquél entonces matemática era prácticamente todo lo que no era ni historia natural, ni medicina, ni cirugia, ni química. El resto, astronomía, cosmografia, navegación, física experimental, gnomónica, relojeria, mecánica, óptica,

15 La falta de uniformidad en las piezas de artillería fue contrarrestada con la publicación de ordenanzas y disposiciones en cada país en las que se prescribía oficialmente las características que debian reunir las nuevas piezas que se fundiesen. Estas nuevas piezas son lo que se conoce como "artillería de ordenanza". Las piezas anteriores no fueron destruidas, sino que siguieron en servicio mientras su estado así lo permitió. En España, a pesar de que las informaciones son contradictorias, parece que ya en 1716 se ordenó la fundición de cañones en un sólo género con cinco calibres diferentes: $24,16,12,8$ y 4 lbras de bala. Para la Marina se destinaban cañones de hierro de dos calibres: 6 y 18 .

16 Véase "Sur le recul des armes à feu" en Hist. Roy. Acad. Sci., 1703. París (1704), pp. 98-100. Más adelante se comprobó la existencia de este desplazamiento lateral del proyectil o deflección. Una primera toma de contancto con la faceta tecnológica e industrial de la artillería de la época, siglos XVII y XVIII, puede conseguirse en DERRY, J.K.; WILLIAMS, Trevor 1.; Historia de la tecnologia. (Desde la Antigüedad 1750), Madrid, S. XXI, $12^{3}$ ed. (1989), (1 ${ }^{3}$ ed. inglesa, 1969); McNEIL, lan (ed.); An Encyclopaedia of the History of Technology; Routledge, London-New York (1990); HALL, A.R., "Military Technology" en SINGER, Charles; HOLMYARD, E.J.; HALL, A.R.; WILLIAMS, Trevor I.; A History of Technology, Vol. III, From the Renaissance to the Industrial Reoolution, c. 1500 - c. 1750. Oxford, Clarendom Press (1957), pp. 347376.

17 Véanse, por ejemplo, CASSIRER, Ernst; opus cit., "II: La naturaleza y su conocimiento en la filosofía de la llustración", pp. 54-112. HANKINS, Thomas; "Historia natural y fisiología", Ciencia e llustración. Trad. española de Alfredo Messa. Madrid, Editorial Siglo XXI (1988) (1 ed. inglesa 1985), pp. 120-169. ROGER, Jacques; Les sciences de la vie dans la pensée française du XVIII siécle. La génération des animaux de Descartes à l'Encyclopédie, Paris, $2^{\mathbf{a}}$ ed. Armand Colin (1971). 
hidrostática e hidrodinámica, construcción naval, fortificación, topografía, artillería, amén del álgebra, la geometria, la trigonometría, el análisis, el cálculo de probabilidades ... todo esto era matemática y como tal lo encontramos en los cursos y compendios, aunque estaba claro que entre el análisis y la construcción naval, por ejemplo, había importantes diferencias que determinaban la división en "matemáticas puras o especulativas" y "matemáticas mixtas".

El análisis experimentaba un incesante enriquecimiento superando la oposición entre newtonianos y leibnizianos, a la vez que reorientaba gradualmente su rumbo alejándose de la geometrización inicial ${ }^{18}$. En el XVIII el cálculo diferencial fue más allá de la mera traducción de problemas geométricos y algebraicos ya conocidos a un nuevo método y con su empleo en la resolución de problemas mecánicos se abrieron campos hasta entonces no conocidos por los geómetras. El método inverso de las tangentes cuajó en el cálculo integral, yendo desde los casos más sencillos a otros más complejos. Incluso algunos supuestamente simples,

18 Una visión amplia de la matemática de la época la encontramos, por ejemplo, en la obra ya clásica de MONTUCLA, J.F.; Histoire des mathématiques, dans laquellé on rend compte de leurs progrès depuis leur origine jusqu'à nos jours; où l'on expose le tableau et le déoeloppement des principales découoertes dans toutes les parties des mathématiques, les contestations qui se son eleoeés entre les mathématiciens, et les principaux traits de la vie des plus celèbres. Edición acabada y editada por Jérôme $\mathrm{DE}$ LA LANDE. Paris, Año X (1802), así como en la más reciente, pero no por ello menos clásica, WÓLF, A.; A History of Science, Technology and Philosophy in the Eighteenth Century. London, George Allen \& Unwin Ltd., 2- ed. (1952), (1 ed. 1938). Véase también BOS, H.J.M.; "Mathematics and rational mechanics" en ROUSSEAU, G.S. y PORTER, Roy (eds.)' The Ferment of Knowledge. Studies in the Historiography of the Eighteenth-Century Science, London, Cambridge University Press (1980), pp. 327 355. El estudio de la mecánica racional en nuestro siglo debe mucho a Clifford Truesdell III. Mucho podrá discutirse acerca de su entusiasmo y su imparcialidad, pero es indudable que ha ejercido un saludable efecto de reanimación en la historia de la mecanica del XVIII. Véanse TRUESDELL, C.; Ensayos de Historia de la Mecánica, trad. española de Juan Carlos Navascués y Enrique Tierno Pérez-Relaño, Madrid, Editorial Tecnos (1975), (1³ ed. inglesa, 1968). TRUESDELL, C. (ed.); Leonhardi Euleri Opera Omnia. Commentationes Mechanicae ad theoriam corporum fluidorum pertinentes. Volumen posterius, edidit Clifford Ambrose Truesdell III. Auctoritate et impensis Societatis Scientiarum Naturalium Helveticae. Lausannae MCMLV. Venditioni exponunt Orell Füssli Turici. Rational Fluid mechanics, 1687-1765 (Editor's introduction to Euleri Opera Omnia II 12). 
como la integración de ecuaciones diferenciales de primer orden o su aplicación a secciones cónicas, podían presentar dificultades insospechadas al aparecer integraciones de orden superior.

En las sociedades científicas y en las reuniones de los salones se discutían las fuerzas vivas y las fuerzas muertas, se defendía o se atacaba a Leibniz, a Maupertuis, a Newton ${ }^{19}$. Se oía hablar del principio de la acción mínima y de la determinación de la figura de la Tierra, se buscaban proporcionalidades entre causas y efectos, entre fuerzas, velocidades y resistencias ${ }^{20}$, y se intentaban formular leyes generales capaces de dar cuenta del mayor número posible de movimientos, desde el movimiento de la luz ${ }^{21}$, el de

19 Basta con una primera lectura de las memorias y experimentos presentados ante las sociedades científicas para hallarse uno inmerso en esta búsqueda incesante de leyes mecánicas, y comprobar cómo se ponía en juego algo más que el talento matemático. Aunque no están directamente relacionados con la balística, merece la pena ver PEMBERTON, Henry; "Concerning an Experiment relating to the force of Bodies in Motion", Phil. Trans., n. 371, p. 57. En la edición aquí utilizada, vol. VI, 1719-1733, pp. 276-280. PEMBERTON, Henry; "An Argument in confirmation of the foregoing Paper", ibidem, n 371, p. 67. Ibidem, pp. $280-281$.

20 DESAGULIERS, J.T.; "An Account of some Experiments to prove that the Force of Moving Bodies is proportionable to their Velocities", ibidem, n. 375, p. 269. lbidem, pp. 281-285. DESAGULIERS; "Animadversions upon some Experiments relating to the force of Moving Bodies, with two new Experiments on the same Subject", ibidem, n. 376 p. 285. lbidem, pp. 285-287. EAMES, John; "A Remark upon the new Opinion relating to the Forces of moving Bodies, in the case of the Collision of non-elastic Bodies", ibidem, n. 396, p. 184. Ibidem, pp. 287-291. EAMES, John; "Remarks upon some Experiments in Hydraulics, which seem to prove that the forces of equal moving bodies are as the squares of their velocities", ibidem, $n$. 400 , p. 343. Ibidem, pp. 292-294. CLARKE, Sam.; "Of the proportion of Velocity and Forces in bodies in Motion", ibidem, n. 401, p. 382. Ibidem, pp. 294-299. La vieja cuestión de qué es lo que permite la continuación del movimiento cuando el móvil ha abandonado el motor renace brevemente en "Sur la continuation du mouvement", Hist. Acad. Roy. Sci., 1701. París (1704), pp. 14-15, sin que de ella pueda extraerse nada útil para la balística.

21 Por ejemplo, "Sur la courbe que décrivent les rayons de la lumière" en Hist. Roy. Acad. Sci., 1702. París (1704), pp. 54-58. También aquí se buscaba determinar la curva descrita por una rayo de luz en su paso a través de la atmósfera. Se partía de que el rayo, al pasar del éter a la atmósfera, experimentaba una diferencia en la densidad del medio que repercutía en su refracción. La Hire defendía que su trayectoria era una cicloide o una epicicloide, dependiendo de cómo se considerasen las capas de la atmósfera. Hubo también un intento de enfocar el movimiento de una bala de mosquete disparada a un recipiente lleno de agua como si se tratase de un rayo de luz sometido a refracción al pasar del aire al agua. CARRÉ; 
cuerpos en caída libre ${ }^{22}$, el de chorros y surtidores ${ }^{23}$, hasta el de los proyectiles; en todos ellos se perseguían denominadores comunes y se intentaba afrontarlos desde una misma perspectiva. Se investigaba cualquier tipo de resistencia al movimiento, no sólo la del aire al paso de proyectiles, con la finalidad no tanto de asentar una teoría determinada como de acumular conocimiento experimental. Era habitual que estos trabajos se limitasen a medir y anotar cuidadosamente todos los factores sin extraer al final ninguna conclusión ${ }^{24}$.

\footnotetext{
"Expériences physiques sur la refraction des balles de mousquet dans l'eau, et sur la résistance de ce fluide" en Mém. Acad. Sci., 1705. París (1706), pp. 211-219. Este tipo de trabajos estaban animados por la esperanza de concluir unas leyes generales válidas para distintos tipos de movimiento, pero la mayoría de las veces no podía extraerse de ellos ninguna conclusión general.
}

22 Por ejemplo, VARIGNON; "Méthode pour trouver des courbes le long desquelles un corps tombant s'approche ou s'éloigne de l'horizon en telle raison des tems qu'on voudra, et dans quelque hypothèse de vîtesses que ce soit" en Mém. Acad. Sci., (1699), pp. 1-12. París (1702) (Retoma el principio de Galileo y los trabajos de Leibniz y los Bernoulli para superarlos, formulando la caída del cuerpo no sólo en relación al horizonte sino con respecto a un punto cualquiera). VARIGNON; "Addition au premier des Mémoires de l'Académie de l'année 1699 touchant la manière de trouver des courbes le long desquelles un corps tombant, s'approche ou s'éloigne de l'horizon, ou d'un point donné quelconque, en telle raison des temps, et dans telle hypothèse des vîtesses que l'on voudra", Mém. Acad. Sci., 1703. París (1705), pp. 140-150. La verificación experimental de la ley galileana de la caída de cuerpos la intentó SEBASTIEN; "Sur une machine faite pour éprouver la proportion de la chûte d'un corps" en Hist. Roy. Acad. Sci., (1699), pp. 116-117. París (1702).

23 Por ejemplo, tanto Mersenne como Torricelli acudieron a experiencias con surtidores y chorros de agua y hallaron que la resistencia del aire tenia efectos apreciables sobre los chorros, aunque no tenían recorridos parabólicos sino más cortos de lo que debieran y cercanos a la vertical.

24 Éste es el caso de un trabajo sobre la resistencia en medios fluidos que Desaguliers presentó a la Royal Society. El experimento consistió en sumergir un pequeño péndulo, cuya lenteja era una esfera de oro, primero en un recipiente lleno de agua, luego en el mismo recipiente lleno de mercurio, y por último en un tubo lleno también de mercurio. Observó cuidadosamente el comportamiento del péndulo en las distintas pruebas, pero no fue más allá de su registro, no extrajo ninguna consecuencia generalizable a otros casos, ni tampoco a la artillería. Véase DESAGULIERS, J.T.; "Experiments relating to the Resistance of fluids", Phil. Trans., March 30th. 1721, n. 367, p. 142. En la edición aquí utilizada vol. VI, 1719-1733, pp. 305-306. Anteriormente también Newton se sirvió de experimentos con péndulos sumergidos en distintos medios para hallar la resistencia de éstos. Véase el Escolio General a la Sección IV, en particular los experimentos descritos en las pp. 520-524 
En los observatorios se seguía trabajando en la astronomía de posición y astronomía física, y los nuevos hallazgos eran la base sobre la que debían contrastarse las líneas maestras de la mecánica celeste newtoniana, la teoría de la gravitación universal, la resolución del problema de los tres cuerpos, la predicción de cometas y la estabilidad del sistema solar. Mientras tanto, en las matemáticas mixtas, la utilización del análisis en la resolución de problemas relativos a las propiedades de las curvas ${ }^{25}$, el estudio de las cuerdas vibrantes, cuestiones de hidrodinámica y una infinidad de problemas de todo tipo, definían el dominio de la mecánica racional, también denominada mecánica analítica, mecánica especulativa o mecánica teórica. Otra manera de definir la mecánica racional, por ejemplo, es hacerlo en contraposición a la mecánica newtoniana y entenderla como la respuesta de los matemáticos continentales a los británicos, o bien contraponerla a la mecánica celeste lo que aproximadamente viene a ser lo mismo, pues durante muchos años la mecánica celeste de la época fue la mecánica newtoniana.

Esta mecánica racional estaba lejos de plantear los problemas habituales en fábricas, industrias y talleres de artesanos. Sus enunciados son ejercicios mentales, planteamientos teóricos que frecuentemente no se dan ni en la naturaleza ni en las obras humanas. En ella las propiedades físicas se idealizan y cuantifican, y la mecánica cobra vida con independencia de si los nuevos descubrimientos tienen aplicación directa. Los dos ejemplos más citados de la utilidad práctica de la mecánica racional son las

de la citada edición española de los Principia.

25 Tanto curvas geométricas (aquéllas cuya naturaleza puede expresarse y determinarse mediante la relación entre ordenadas y abscisas, siendo éstas magnitudes finitas) como mecánicas o trascendentes (aquéllas cuya naturaleza no puede expresarse de esta forma pues no existe una relación según reglas entre ellas). Las secciones cónicas son curvas geométricas, mientras que la cicloide, la espiral, la catenaria, la braquistocrona, la cáustica etc. son curvas mecánicas. Véase, por ejemplo, "Sur les vîtesses des corps mûs suivant des courbes" en Histoire de l'Académie Royale des Sciences. Année MDCCIV. Paris (1706), pp. 104-116. 
aportaciones de Leonard Euler al estudio de la construcción naval y al de la artillería. Nada puedo decir respecto a la construcción naval, pero en cuanto a la artillería se verá cómo los comentarios de Euler a los New Principles of Gunnery de Benjamin Robins, y algún otro trabajo propio presentado a la Academia de Ciencias de Berlín, estaban lejos de poder ser puestos en práctica inmediatamente por los artilleros. Así pues, incluso algunas de las cuestiones aparentemente más prácticas de la mecánica racional distaban mucho de serlo efectivamente a corto plazo. Para comprobarlo basta con repasar los trabajos presentados en sociedades y academias, en donde tras títulos que prometen una cierta utilidad práctica hay hipótesis y teorias generales desarrolladas en el supuesto de condiciones inverosímiles en la realidad ${ }^{26}$. No le falta razón a Truesdell cuando afirma que la mecánica racional mantiene con la realidad física la misma relación que con ella pueda mantener la geometria, es decir, bastante escasa. Tras sus estudios, la mecánica racional se ha revelado como una ciencia axiomática, no experimental. La mecánica racional que exponía Montucla es, en manos de Truesdell, mecánica racional axiomáti$\mathrm{ca}^{27}$.

26 Por ejemplo, l'HOPITAL, "Méthode facile pour trouver un solid rond, qui étant mû dans un fluide en repos paralellement à son axe, rencontre moins de résistance que tout autre solide, qui aïant même longueur et largeur se meuve avec la même vitesse suivant la même direction", Mém. Acad. Sci., Paris (1969), pp.107112 , o bien, VARIGNON, "Manière de discerner les vîtesses des corps mûs en lignes courbes, de trouver la nature ou l'équation de quelque courbe que de soit engendré par le concours de deux mouvements connus, et réciproquemente de déterminer une infinité de vîtesses propres deux à deux à enfendrer ainsi telle courbe qu'on voudra, et même de telle vitesse qu'on voudra suivant cette courbe". Mém. Acad. Sci., Paris 1704 (1706), pp. 286-306.

27 Truesdell deshace el espejismo de la mecánica de Newton reinante hasta la aparición de la Mécanique analitique de Lagrange (1788) y de la Mécanique céleste de Laplace (1799-1825). Distingue entre "leyes de Newton" (aquéllas que de hecho fueron enunciadas por él) y "ecuaciones newtonianas" (las que suelen presentarse en los libros de física como tales), desenmascara el orígen de los dos enunciados que hoy se conocen como 'principio de d'Alembert' y que, según él, en realidad son obra de Euler y Lagrange, rastrea el significado de conceptos físicos a partir del uso que de ellos se hace en la práctica y no de las definiciones que aparecen en los libros, estudia directamente los escritos de Newton, Euler, Lagrange, los Bernoulli, 
El problema balístico es un caso particular, y no de los menos espinosos, de esta mecánica raciona ${ }^{28}$. A primera vista su resolución parece ser de una utilidad pública semejante a la que tenia la determinación de la longitud en el mar, y por tanto debería haber despertado el interés de gobiernos y sociedades científicas pero, sorprendentemente, no fue objeto de un premio en una academia de este tipo hasta 1782. Aunque pueda parecer extraño, a juzgar por la escasa atención que se le ha dedicado en la historia en general y la pobre bibliografía centrada en este período, se trata de un problema de rango secundario, tratado ocasionalmente por numerosos matemáticos célebres sin pretensiones de aplicar sus teorías en la practica ${ }^{29}$. La dificultad en la resolución del problema balístico ha sido puesta de manifiesto repetidamente y se ha señalado que tal vez nunca llegue a ser resuelto con toda exactitud $^{30}$. Ninguna de las numerosas tablas balísticas que se han

y busca en ellos las formulaciones y definiciones originarias. Entre otras cosas, pone de manifiesto la insuficiencia de las leyes de Newton para dar cuenta de tres tipos de sistemas mecánicos: los de cuerpos rígidos, cuerpos flexibles y cuerpos elásticos, así como la incapacidad de sus discípulos y rivales para resolver los problemas relativos al movimiento de cuerpos deformables.

28 La percepción que a mediados de siglo se tenía de él dentro del panorama general del conocimiento queda patente en la clasificación que aparece al inicio de la Encyclopédie de d'Alembert y Didenot, la cual se basaba en la clasificación de Bacon. En ella aparecía incluida junto con la dinámica propiamente dicha, y la hidrodinámica en la dinámica, la cual junto con la estática constituía la mecánica. Ésta última se encuadraba con la astronomía geométrica, la óptica, la acústica, la pneumática y el análisis de probabilidades en la matemática mixta. DIDEROT y D'ALAMBERT; Encyclopédie ou dictionnaire raisonné des sciences, des arts et des métiers. Paris (1751-1772). Edición facsímil de Franco Maria Ricci, Parma.

29 No hay que dejarse deslumbrar por frases del tipo de "Avec la chute des graves, les oscillations du pendule et les mouvements planétaires, l'étude de la trajectoire des projectiles est de celles qui ont les plus contribué à l'établissement des principes de la mécanique rationenelle", d'OCAGNE; opus cit.. De hecho, las fechas de aparición de los primeros tratados de balística como tal, no de artillería, datan del ultimo cuarto del XVIII.

30 Por ejemplo, DIDION; Traité de balistique (1847), "... aucune méthode rigoureuse $n^{\prime} a \mathrm{pu}$ jusqu'ici exprimer une relation finie entre les angles de projection, la vitesse initiale et l'amplitude du jet; peut-être même, ne pourra-t-on jamais résoudre cette question dans toute sa rigueur...", p. 80. Años después seguiremos encontrando expresiones del mismo tipo, por ejemplo, en MATA, Onofre; "La ley de la resistencia del aire según la termodinámica" en Memorial de Artilleria, serie IV, t. II, Madrid (1894), afirma que "Los resultados experimentales 
construido desde los tiempos de Alava $^{31}$, Collado $^{32}$ y Ufano ${ }^{33}$ hasta las de Euler ${ }^{34}$ o Gravenitz (éste último casi mediado el siglo $X I X)$ ha podido dar cuenta satisfactoriamente de los múltiples factores que intervienen en la formulación y resolución del problema.

La trayectoria parábolica, que ya coexistió con la trayectoria logarítmica postulada por Huygens para la resistencia directamente proporcional a la velocidad del móvil ${ }^{35}$, convive ahora con la trayectoria hiperbólica, consecuencia de la ley cuadrática de la resistencia que se desprende de la teoría newtoniana, profundizada y desarollada por Johann y Nicolás Bernoulli, Jakob Hermann, Varignon etc.. Sin embargo, ninguno de estos trabajos, a pesar de su indudable mérito, marca un punto de inflexión definitivo en la historia y el origen incuestionable de la artillería moderna ${ }^{36}$.

han sido posteriormente (a Mayewski, Bashfort y Krupp) el punto de partida para expresar algebraicamente la ley de la resistencia del aire, ya que hasta el presente no se ha podido establecer directamente de una manera teórica", pp. 5-6.

31 ALAVA Y VIAMONT, Diego de; El perfecto capitán instruido en la disciplina militar, y nueva ciencia de la artilleria, Madrid (1590).

32 COLLADO DE LEBRIJA, Luis; Platica manual de artigleria. Venecia (1586).

33 UFANO, Diego; Tratado de la Artillería y uso della, platicado por el capitán Diego Ufano en las guerras de Flandes. Bruselas (1613).

34 EULER; opus cit.

35 Véanse HALL, A.R.; Ballistics in the seventeenth Century. A Study in the Relations of Science and War with Reference principally to England. Cambridge, University Press (1952). Capitulo V, "The Resistance of Air", pp. 102-129. DUGAS, R.; La mécanique au XVIle siècle, Chapitre XV "Dynamique galiléenne, mouvement en milieu résistant et art de jetter les bombes", pp. 521-555. Neuchatel, Ed. du Griffon (1954).

36 El empleo de la expresión "artillería moderna" sólo está plenamente justificado para la artillería del siglo XIX, cuando los cañones de avancarga, los proyectiles esféricos y la polvora negra graneada han dejado su sitio a las piezas de retrocarga, los proyectiles ojivales y los nuevos tipos de pólvoras y explosivos. La formación de un comité de artillería en Metz en 1833 para la investigación de las leyes balisticas y la convocatoria en esa misma época de un premio de la Academia de Ciencias de París, ganado por Didion, Piobert y Morin, bien pueden saludarse como un hito en la escasa investigación balística patrocinada por instituciones nacionales. Pero también hay quien más tajantemente expone la historia de la balística como si entre 1742, fecha de la publicación de los New Principles of Gunnery de Robins y 1897, año en que E. Mach edita Popular Scientific Lectures, en donde se incluía "On some phenomena attending the flight of 
A partir de Newton y Robins se suceden, por una parte, una serie de valiosas aportaciones matemáticas en las que, sirviéndose de diferentes métodos, el enunciado del problema balístico se generaliza cada vez más (cuadraturas, series convergentes, sustitución de expresiones no integrables por otras que sí lo son etc., debidos a los Bernoulli, Euler, Lambert, Borda etc.), y por otra parte, los planteamientos de tipo físico-matemático acompañados de experiencias de artillería (Robins, Hutton). Ambos tipos de trabajos coexisten simultáneamente y no puede decirse que en un momento determinado un procedimiento se imponga al otro. Gracias a ambos cada vez se tienen en cuenta un mayor número de factores $e$, inevitablemente, el problema adquiere un mayor grado de complejidad.

2.- Salvando el abismo: el péndulo balístico. Determinación experimental de la velocidad del proyectil

A) Benjamin Robins (1742)

Matemáticos y artilleros estaban condenados a entenderse, aunque ésta no fuera una cuestión tan acuciante como hoy en día pudiera parecer. Dejemos a un lado el planteamiento más puramente analítico y el más estrictamente práctico y centrémonos en el desarrollo matemático-experimental, es decir, en la resolución de cuestiones de carácter físico utilizando los mejores conocimientos matemáticos entonces disponibles junto con aparatos expresamente diseñados para la experimentación física, como sucede en la determinación de la velocidad del proyectil mediante el péndulo balistico ${ }^{37}$.

projectiles" no se hubiera hecho nada digno de mérito. Éste es el caso de SEEGER, R.J.; "On Aerophysics Research" en American Joumal of Physics (1951) pp. 459-469.

37 He desarrollado un primer acercamiento al estudio de ambas comunidades en mi tesis doctoral Ciencia $e$ institución militar en la España ilustrada. El caso de la artillería. U.N.E.D. Departamento de Antropologia, Lógica, Filosofía de la Ciencia e Historia de la Ciencia, 1993. 
La determinación del proyectil tiene un doble valor, en sí misma puesto que se trata de uno de los factores que intervienen en el problema balístico (la resistencia del aire es proporcional a su cuadrado, según Newton) pero, además, era una de las formas de evaluar la fuerza de distintas pólvoras, asunto éste último que preocupaba a los artilleros bastante más que el cálculo de la resistencia del aire.

Para la medición de la fuerza de las pólvoras era habitual la utilización no sólo de instrumentos basados en péndulos balísticos, sino también de morteretes y probetas. Los primeros eran pequeños morteros fijados a planchas y con una elevación fija de $45^{\circ}$ utilizando una pequeña bala de bronce. Se realizaban disparos con distintos tipos de pólvora y los alcances logrados se tomaban como índices de la potencia de las pólvoras. Entre las probetas la más extendida era la que se conocía como probeta d'Arcy, por ser descrita por vez primera por Patrice d'Arcy en su Essai d'une théorie d'artillerie (1760), y que venía a ser un péndulo balístico en el que se suspendía un pequeño cañón ${ }^{38}$. Por todo ello, las experiencias que Robins publicó en 1742 en New Principles of Gunnery llamaron la atención tanto de los matemáticos como de los artilleros, al menos, de aquellos artilleros que disponían de la adecuada formación e información.

Benjamin Robins, ingeniero militar e hijo de padres cuáqueros, demostró su escasa inclinación por la paz tanto en su ardorosa y ruda defensa de Newton frente a los matemáticos continentales como en su interés por la balística y la fortificación ${ }^{39}$. Su nombre

38 Sobre estos instrumentos véase HIDALGO CAMARA, E.; "El arte de fabricar póloora (1800) de Tomás de Morla". Congreso Internacional Louis Proust. Teoría, práctica y difusión científicas en la química europea del S. XVIII. Segovia, Mayo de 1992. Próxima publicación.

39 Fue designado "Engineer General" de la la East India Company en 1749, y en la India transcurrió su último año de vida. Entre otros cometidos se le encargó supervisar el entrenamiento adecuado para el uso de la artillería, la elección de la munición, redacción de numerosos inventarios, levantamientos de planos de fuertes y puertos, etc. Según $W$. Johnson su tarea era muy similar a la encomendada a los capitanes de barcos holandeses y españoles, y los resultados se mantenían en el 
ya era conocido en Londres por su participación en una disputa matemática con James Jurin entre 1735 y 1737, la cual quedó registrada en Republic of Letters. ${ }^{40}$.

El libro que le proporcionó un lugar en la historia de la balística fue New Principles of Gunnery ${ }^{41}$, en donde la importancia de la descripción del primer péndulo balístico para medir experimentalmente la velocidad de los proyectiles quedó patente al estimar Euler que su planteamiento newtoniano del problema balístico, y sus experiencias con el péndulo, eran lo suficientemente valiosas como para dedicarse personalmente a su traducción y comentario ${ }^{42}$, consagrando así definitivamente el nombre de Robins. Ciertamente el texto original inglés fue enriquecido muy notablemente por los comentarios de Euler, cuyas notas incluso son más extensas que el texto original al que comentan. El resultado final constituye, en palabras de Truesdell, "la primera obra científica sobre artillería", quien se refiere a ella como "el gran

mayor de los secretos. Un caso más de los habituales "militares-espías-científicos" de la época. Sobre la vida y obra de Benjamin Robins véanse los diversos estudios que sobre él ha publicado Johnson, por ejemplo, JOHNSON, W., F.ENG.,F.R.S.; "Benjamin Robins, F.R.S. (1707-1751): New details of his life", Notes Rec. Soc. Lond., 46 (2), 235-252 (1992).

40 Robins utilizaba en el cálculo de fluxiones la idea de un valor límite al que una variable puede aproximarse pero sin llegar nunca a alcanzarlo. Jurin defendía que esos límites inalcanzables eran valores que en la naturaleza se alcanzan en un tiempo finito. En respuesta a los ataques de Berkley contra el análisis, Robins publicó un Discourse concerning the nature and certainty of Sir Isaac Newton's Method of fluxions, Londres (1735). Montucla comenta que en la traducción que Buffon hizo del tratado de las fluxiones de Newton señalaba que Robins había dicho que Newton se equivocó al idear su principio, pero Montucla no halla motivo para la observación de Buffon y estima que el trabajo de Robins sobre las fluxiones es sólido y satisfactorio, aunque tal vez fuera excesivamente duro al enjuiciar a Johann Bernoulli y a Euler. MONTUCLA, opus cit., Part. V, Liv. I, pp. 118-119.

41 New Principles of Gunnery (1742). La otra gran obra de Robins, Mathematical Tracts, 2 vols., Londres (1761), es una recopilacion de sus trabajos incluido los New Principles..., junto con la controversia sobre la vis viva y diversas memorias y artículos.

42 Neue Grundsätze der Artillerie, aus dem Englischen des Herm Benjamin Robins übersetzt und mit vielen Anmerkungen versehen. Berlin (1745). 
tratado sobre balística de Euler' ${ }^{\prime 43}$. Sin duda alguna, la obra resultante del original de Robins y de los comentarios de Euler es un punto de referencia inexcusable en el posterior desarrollo de la balística matemática ${ }^{44}$. Además, rebasa los límites de ésta para entrar de lleno en el estudio general de la resistencia de los fluidos sobre los cuerpos en movimiento, en la línea más clásica de la mecánica racional.

Hasta ese momento la balística newtoniana era una cuestión teórica, que se correspondía con la realidad dentro de unos márgenes imprecisos. Robins toma de Newton lo esencial de su teoría (proporcionalidad cuadrática de la resistencia y trayectoria hiperbólica) y se dispone a determinar experimentalmente esos márgenes de aplicación. Acompaña sus experiencias físicomatemáticas con hipótesis acerca del comportamiento de la pólvora, basadas a su vez en prácticas bien conocidas entre los artilleros e ingenieros de la época, pero poco afortunadas. En Robins no encontramos la creación matemática original de altos vuelos que hallamos después en Euler, sino una reflexión práctica muy competente sobre la hipótesis cuadrática de la resistencia. Su obra proporciona un instrumento sumamente valioso para acercar la teoría a la práctica, contribuyendo a que la utilidad de la primera fuera cada vez más difícilmente discutible.

Sus conocimientos en materia militar se revelan en el discurso preliminar de los Nuevos principios..., en donde hace un repaso de la historia de la fortificación, la de la pólvora y la de las piezas de

43 "... Euler's great treatise on ballistics -for the annotations which he added to his translation of Robin's little budget of rules, experiments and guesses transformed it into the first scientific work on gunnery", p. XXXVחI, Leonhardi Euleri Opera Omnia, Sub auspiciis societatis scientiarum naturalium Helveticae. Series secunda, Opera Mechanica et Astronomia. Volumentertium decimum, Lausannae MCMLV'.

44 Aquí me he servido de una traducción francesa de la edición comentada por Euler, Nouveaux principes d'artillerie, de M. Benjamin Robins, commentés par M. Leonard Euler, traduit de l'allemand avec des notes, par M. Lombard, professeur royal aux Écoles d'Artillerie à Auxonne. Dijon (1783). Parece ser que también Lambert, según se indica en las advertencias a la segunda edición del Tratado de artillería de Tomás de Morla (1816), hizo una traducción al francés de esta obra de Robins. 
artillería, para a continuación citar a los autores, a su juicio, más importantes de la historia de la balística: Tartaglia, Busca, Collado, Ufano, Simienowicz, Bourne, Eldred, Anderson, Galileo, Blondel, Halley y Newton. Meter a Newton en el mismo saco que a los demás es un desliz que no cuadra bien con su ardor newtoniano. No es de extrañar que Euler, cortésmente, le reprochara el olvido de algunos significativos autores continentales, como los Bernoulli y Hermann, y señalara su débil conocimiento de la teoría de pólvoras, en la que tan sólo citaba a de la Hire quien, por otra parte, no era el autor más fiable ${ }^{45}$. Este discurso preliminar concluye diciendo: "Concluons que jusqu'à présent la science de l'artillerie n'a porté que sur de faux principes \& que sa théorie, dans l'état où elle est aujourd'hui, est inutile \& défectueuse dans la branche la plus importante ${ }^{146}$.

Los Nuevos principios ... fueron presentados a la Royal Society y la correspondiente recensión y crítica apareció publicada en las Philosophical Transactions ${ }^{47}$. Aunque extensa, esta recensión se centra en el primer capitulo de la obra y en las proposiciones del segundo relativas comportamiento de las pólvoras. En él se presentan las experiencias con el péndulo en función de la investigación de la fuerza absoluta de éstas. El comentarista se detiene en la exposición de los presupuestos de los que parte Robins (inflamación instantánea y completa de la pólvora antes de que la bala abandone la pieza) y, en particular, intenta conciliar sus tesis con los resultados obtenidos por una comisión designada

45 La Dissertatio de efferoescentia et fermentatione de Johann Bernoulli (1690) y la Hydrodynamica de Daniel Bernoulli (1738) son dos obras de lectura obligada en la teoría de pólvoras. Los Nuevos principios ... contienen tanto proposiciones relativas a pólvoras como al movimiento de proyectiles.

46 ROBINS, Benjamin; opus cit., p. 33.

47 "An Account of a Book intituled New Principles of gunnery, containing the Determination of the Fonce of Gunpowder; and an Investigation of the resisting Power of the Air to swist and slow Motions; by B.R. F.R.S. as far as the same relates to the Force of the Gunpowder". Read April 14. and 21.1743. Philosophical Transactions (from the Year 1719 to the Year 1733). Abridged and disposed under General Heads, vol. VIII (1732-1744) by John Eames F.R.S. and John Martin F.R.S., London. Pp. 259-270. 
por la Royal Society para investigar esos mismos puntos. Es muy posible que el libro estuviera ya en la calle cuando esta comisión comenzó sus investigaciones y, aunque no hubiera sido así, Robins exponía sus conclusiones a partir de sus propias experiencias. El desacuerdo entre ambos estudios era aun más llamativo por la proximidad de las fechas, de hecho ambos trabajos aparecen publicados en las Philosophical ... uno a continuación del otro. El autor de la recensión explica la posición de Robins alegando que la cantidad de pólvora que queda sin inflamar es despreciable en el tipo de arma empleado por él. Ahora bien, a la vista del rumbo que tomaría la balística con las anotaciones de Euler, en las que ningún factor es en principio despreciable por mínimo que parezca, este tipo de decisiones acerca de qué es importante y qué no lo es resultan cada vez más arriesgadas.

El libro se divide en dos capítulos que, a su vez, se subdividen en proposiciones. Euler procede en sus comentarios siguiendo más - menos una misma pauta: primero demuestra matemáticamente, utilizando el cálculo diferencial e integral, lo que Robins expone geométricamente, añadiendo únicamente la conversión de las unidades de medida inglesas a las alemanas. A continuación revisa, critica y completa, si es necesario, este planteamiento y hace las reflexiones oportunas, bien de carácter práctico para la artillería, bien matemáticas y relativas a la resistencia que ofrecen los fluidos al movimiento de cuerpos. Euler hace gala aquí de un razonamiento físico-matemático más fino y sutil que el de Robins, y detecta factores que intervienen en el tiro balístico en los que éste no habia reparado. Otra cosa es que estos elementos sean o no decisivos en el cálculo de la resistencia de los fluidos ${ }^{48}$.

48 Con anterioridad a esta ocasión Euler no se había ocupado directamente de - cuestiones balísticas, pero sí de aspectos de las propiedades del aire, tales como su elasticidad, que están relacionados con la determinación de la resistencia. Así lo hace en un trabajo publicado en las Memorias de la Academia de San Petersburgo en 1727. Véase "Extrait d'une dissertation de Mr. Euler sur l'explication des phénomenes de l'air." Tomo II, Commentarii Petropolitanae ... (1727). Un extracto de este trabajo puede verse en ROBINS, opus cit., pp. 532-538. Brevemente, Euler supone que el aire está formado por una infinidad de burbujas diminutas, cada una 


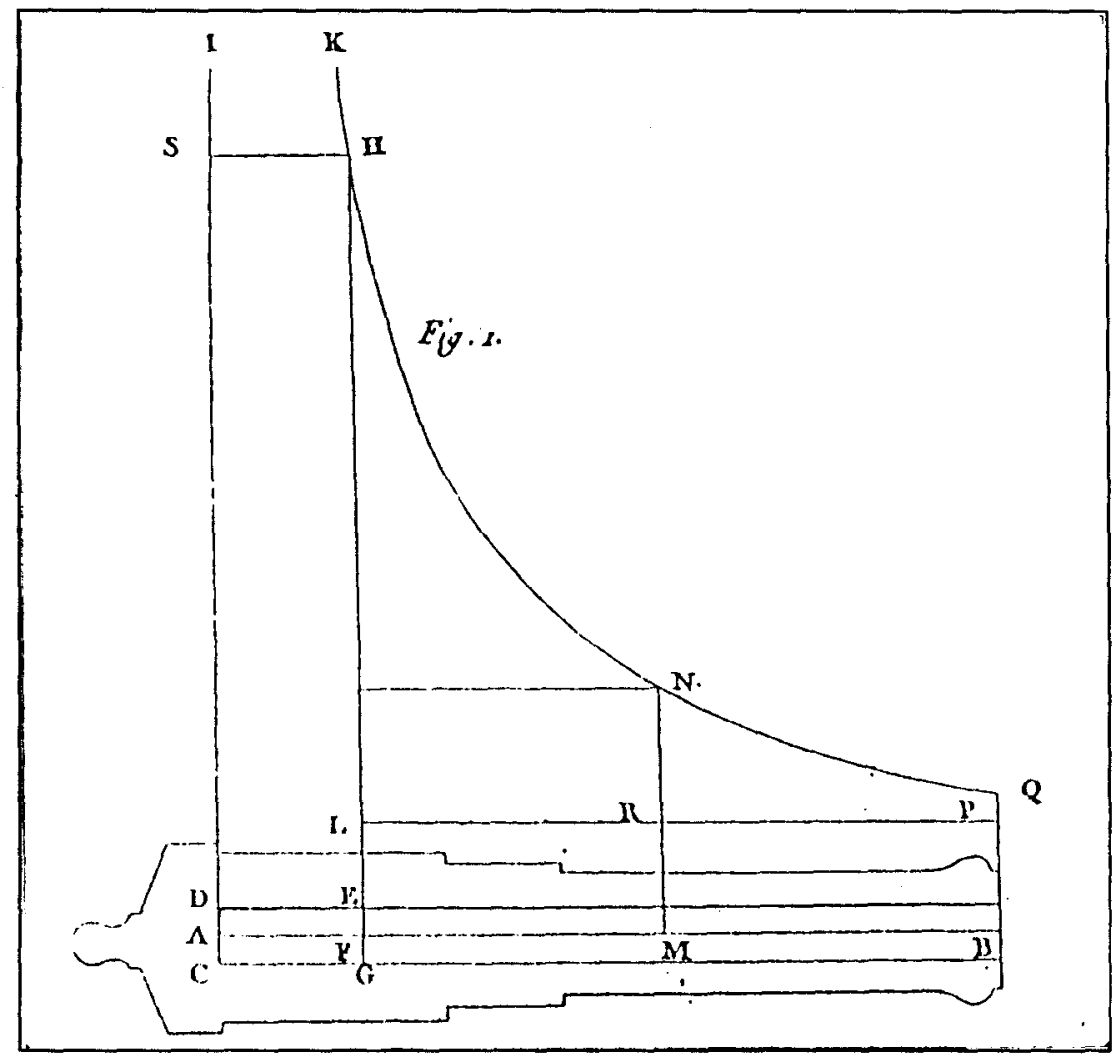

figura 1

de ellas envuelta por una película acuosa. Estas burbujas contienen una materia sutil que se mueve circularmente y tiende continuamente a esparcir las burbujas por su fuerza centrífuga. Este movimiento se mantiene y se acelera debido a otra materia aun más sutil, que penetra y atraviesa libremente todos los poros. Dicha estructura explica que el aire sea susceptible de una condensación limitada y que, si no fuera por la acción de la gravedad, se extenderia al infinito. Euler supone que la velocidad con que la materia sutil circula dentro de cada burbuja es igual en todas las partículas, pues de lo contrario la fuerza centrífuga de las burbujas mayores sería tambien mayor, lo cual es contrario a la experiencia. Así pues, considera que la elasticidad del aire es igual a la fuerza centrifuga de las burbujas $y$, en igualdad de condiciones, proporcional a la altura desde la que debería caer un cuerpo para adquirir la velocidad con la que la materia sutil circula dentro de las burbujas de aire. 
En el primer libro, tras varias proposiciones relativas a la teoría de pólvoras, expone la determinación teórica y experimental de la velocidad inicial del proyectil. Robins sostiene que la inflamación de la carga de la pólvora es instantánea y no sucesiva, como afirmaba Bélidor, lo cual supone que la acción de la bala cesa en el instante mismo en que ésta sale de la pieza, y que la pólvora se inflama por completo y se convierte en fluido elástico antes de que la bala sea sacudida de manera sensible.

Véase la figura 1, en donde se aprecia que la fuerza que actúa sobre la bala sigue también una ley hiperbólica. Sean: $A B$ eje del cañón, $A$ fondo del ánima, $B$ boca del cañón, $C D$ diámetro del ánima, DCGE espacio ocupado por la carga de pólvora, EG superficie en la que la bala toca a la carga de pólvora, $F B$ dirección en que es arrojada la bala, $F H$ perpendicular al eje $A B, A I$ paralela a $F H, K H N Q$ hipérbola descrita entre las asíntotas $A I$ y $A B$ que pasa por el punto $H, F H$ representa la fuerza que actúa sobre la bala en $F, M N$ ordenada por la que se representa la fuerza que actúa sobre la bala cuando llega al punto $M$ y la empuja en la dirección $M B$.

Según Robins, cuando el fluido elástico de la pólvora se dilata hasta llegar a $M$, su elasticidad es a la elasticidad que tenía cuando estaba encerrado en el espacio $A F$ como la línea $A F$ es a $A M$, o como $M N$ es a $F H$. Si $F H$ representa la fuerza que actúa sobre la bala en $F$, entonces $M N$ representa la fuerza que actúa en $M$. En la figura, el espacio hiperbólico $F H Q B$ representa el cuadrado de la velocidad con la que es arrojada la bala fuera del cañón. La superficie rectilínea $L P F B$ representa el cuadrado de la velocidad con la que la misma bala sería arrojada si la fuerza que la empuja fuera constante e igual al peso de la bala. La velocidad que la bala tendría en $B$, si fuera empujada a lo largo de $F B$ por una fuerza constante e igual a su peso, sería igual a la que habría adquirido el caer libremente desde una altura igual a $F B$. $Y$ la velocidad que la bala habría adquirido al caer desde una altura igual a la línea $F B$ es a la velocidad con la que es arrojada como la raíz cuadrada 
del espacio rectilíneo $F L P B$ es a la raíz cuadrada del espacio hiperbólico $F H Q B^{49}$. Es decir,

$$
\frac{v_{s}^{2}}{v^{2}}=\frac{F L P B}{F H Q B}
$$

en donde $v_{s}^{2}$ es la velocidad del proyectil que cae desde una altura igual a $F B^{50}$.

Partiendo de esta dsmostración rigurosamente newtoniana, y por tanto fuertemente geométrica, Euler pasa a dar la suya analíticamente $e^{51}$. Incluye además el diámetro de la bala (Robins daba el diámetro del ánima $d$, pero no el de la bala; la diferencia entre ambos diámetros es lo que se denomina "viento de la bala"),

49 ROBINS; opus cit., "Capítulo I. Proposición VII: Connoissant la longueur et le calibre d'une pièce de canon, le poids du boulet, la charge de la poudre et sa force élastique, au premier instant de l'inflammation, déterminer la vîtesse avec laquelle la boulet est chassé hors du canon", pp. 75-108.

50 Se establece una proporción directa entre estas áreas y los cuadrados de las velocidades, que representan la fuerza aplicada sobre el proyectil. Sabemos que un área hiperbólica es igual a

$$
\int_{x_{1}}^{x_{2}} \frac{d x}{x}=\ln \left(\frac{x_{2}}{x_{1}}\right)
$$

luego aquí, el área $F H Q B$ es igual a

$$
\int_{A F}^{A B} \frac{d x}{x}=\ln \left(\frac{A B}{A F}\right) \approx v^{2}
$$

y el área $F H M N$ lo es a

$$
\int_{A F}^{\mu M} \frac{d x}{x}=\ln \left(\frac{A M}{A F}\right)=v_{M}^{2}
$$

Por lo tanto, en cada punto el cuadrado de la velocidad es proporcional al logaritmo neperiano de la distancia recorrida.

51 Cortesmente Euler comentaba a propósito: "L'auteur détermine ici la vîtesse avec laquelle un boulet est chassé $\mathrm{d}^{\prime}$ 'une piece de canon, et il emploie à cet effet une méthode purement géométrique, en faveur, sans doute, de ceux à qui les nouveaux calculs ne sont pas familiers. Mais pour ceux de nos lecteurs qui connoissent l'usage des formules algébriques, nous allons donner une solution analytique du même problème", "Premier remarque" de Euler a la "Proposition VII", p. 85. 
y su peso específico $P$ (siendo el peso específico del agua igual a 1). En principio, acepta el valor que da Robins para la elasticidad de la pólvora (1.000 veces superior a la elasticidad del aire). Así tenemos: la elasticidad de la pólvora en $A F$ (en el primer instante de la inflamación) es $m$ veces mayor que la elasticidad del aire (según Robins 1.000 veces superior) ${ }^{52}, F M$ (espacio recorrido por la bala cuando ha llegado al punto $M$ ) es igual a $x$, y la velocidad de la bala en $M$ (igual a la que habría adquirido en caída libre) es igual a $v$.

Euler, tras establecer que la velocidad de la bala en el punto $M$ es

$$
v=\frac{48 m b}{P d} \log _{e} \frac{b+x}{b}
$$

donde $\log _{e}$ es el logaritmo hiperbólico ${ }^{53}$, corrige a Robins, quien introdujo la longitud $A F(=b)$ entre las cantidades que intervienen en la determinación de la velocidad. Repite también uno de los ejemplos de disparo de una bala de plomo con un fusil, y halla el mismo resultado, con la única salvedad de que Euler los expresa en pies renanos y Robins en pies de Londres.

Hasta aquí la corrección que Euler hace de la primera resolución geométrica que Robins ofrece del problema balístico, en la que no se revelan errores de cálculo serios. Ahora bien, es evidente que este planteamiento adolece de dos carencias importantes: se ha prescindido de la presión atmosférica ${ }^{54}$ y de la resistencia del aire.

A pesar de algunos defectos, el planteamiento newtoniano de

52 Cuando todo $A F$ está lleno de pólvora. Si $A F$ sólo está parcialmente lleno $m$ disminuirá proporcionalmente.

53 Los logaritmos hiperbólicos, nombre dado en la época a los logaritmos neperianos se deducían de los logaritmos ordinarios de las tablas multiplicando éstos por 2,302585, que era el logaritmo hiperbólico de 10.

54 La presión atmosférica se consideraba igual a una columna de agua de 32 pies de altura. 
Robins casaba bien con los resultados de sus experiencias. Euler lo explica alegando que, de alguna manera, el tipo de pólvora empleada propiciaba la compensación de errores. El acuerdo entre teoría y práctica se daba sólo en los casos en que el incremento de la fuerza aceleradora, resultado de la inflamación sucesiva, se compensaba con la pérdida de fluido a través del fogón y el viento de la bala. Euler está dispuesto a admitir que esta compensación puede producirse en el tipo de pólvora de la que Robins se ha servido (pólvora graneada y no polvorín) ${ }^{55}$. Pero de ninguna manera está dispuesto a ceder en la estimación de la elasticidad de la pólvora y defenderá la valoración de Daniel Bernoulli, 10.000 veces mayor que la presión atmosférica.

De lo que ahora se trata es de averiguar experimentalmente la velocidad de los proyectiles. Aquí es donde entra en juego el péndulo balístico ${ }^{56}$. Éste se basa en el principio de conservación de la cantidad de movimiento. Si $m$ es la masa del proyectil, $M$ la masa del péndulo, $V$ la velocidad con que se mueve el péndulo y $v$ la velocidad de la bala en el momento del impacto, entonces

$$
\begin{aligned}
& m v=(M+m) V \\
& v=\frac{(M+m) V}{m}
\end{aligned}
$$

Véase la figura 2. $A B C D$ es una especie de caballete a cuyas patas $B$ y $C$ están unidas dos brazos $R$ y $S$, capaces de soportar el péndulo $E F G H I K$ por medio del travesaño $E F$, que es el eje del movimiento. Este péndulo debe poder moverse libremente y oscilar sobre dicho travesaño. El cuerpo central del péndulo es de

55 Lo habitual era que en las piezas de artillería, cañones y morteros, se utilizase pólvora graneada, y que en las armas de fuego de mano, especialmente en las que se empleaban en la caza, se utilizase polvorin, una mezcla más fina y molida.

56 "Proposition VIII: Trouver, par le moyen de l'expérience, avec quelle vîtesse une balle se meut, à une distance quelconque du canon", ROBINS, opus cit., pp. 109139. 
hierro y su parte inferior es más ancha que el resto. Ésta se halla recubierta por una plancha de madera GHIK sujetada por algunos tornillos. Por debajo del péndulo hay otro travesaño $O P$, unido a las patas $B$ y $C$, en cuyo centro se fija un instrumento $M N V$ semejante a la punta de una pluma de dibujo, es decir, con dos láminas de acero que pueden alejarse o aproximarse entre sí por medio de un tornillo. Finalmente, a la parte inferior del péndulo se le ata una cinta $L N$ que se hace pasar entre las dos láminas metálicas y que después cae libremente al suelo en $W$.

Conociendo el peso del péndulo, la situación de su centro de gravedad y la de su centro de oscilación, podrá hallarse la distancia de estos dos puntos al eje de suspensión. Con lo anterior, más el peso y la velocidad de la bala se podrá determinar el movimiento que el péndulo recibe por el choque de una bala que impacta en él en un punto dado. Si el péndulo está en reposo antes del choque, podrá hallarse la extensión de la primera vibración después del choque o su alejamiento. $Y$ recíprocamente, conociendo la extensión de la vibración, el peso de la bala y el punto en que impacta en el péndulo puede determinarse la velocidad de la bala en el momento del choque. Estas experiencias se realizaron con fusiles y no con cañones por razones obvias. Un fusil es mucho más fácil de manejar, y un dispositivo de este tipo no hubiera soportado el impacto de una bala de cañón. Charles Hutton se encargaría de hacer en Woolwich este mismo tipo de experiencias con piezas de gran calibre a partir de 1775 .

Una experiencia de este tipo, aparentemente aséptica, estaba condicionada por una multitud de factores estrictamente prácticos. Por ejemplo, el bloque de hierro se recubre con la plancha de madera porque si la bala impactara directamente sobre el metal se rompería y sus pedazos se incrustarían en el caballete; el peso del péndulo y el espesor de la plancha de madera deben ser proporcionales al grosor de la bala; como la plancha de madera sólo puede sujetarse al bloque de hierro mediante tornillos metálicos, y la cabeza de éstos siempre quedará sin cubrir, puede suceder que la bala impacte sobre ellos y se rompa en pedazos; si la carga 


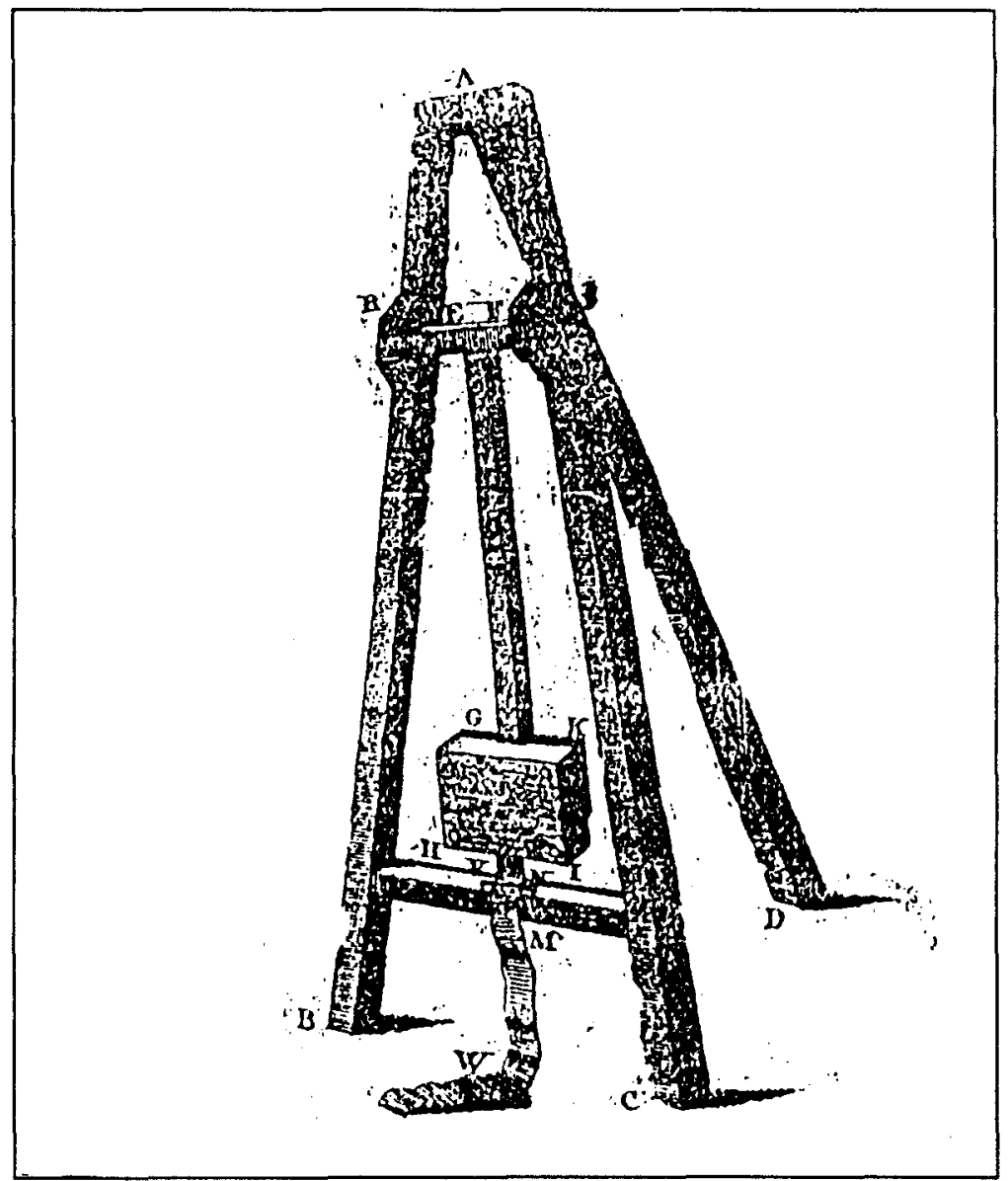

Figura 2

de pólvora es demasiado débil y la bala no alcanza una velocidad de 400 a 500 pies/sg (de 121,92 a $152,4 \mathrm{~m} / \mathrm{sg}$ ), la bala no se incrustará en la plancha sino que será rechazada con una fuerza tanto mayor cuanto más dura sea la madera; hay que fijar sólidamente el cañón del fusil para así evitar las sacudidas, los que mejor se prestan a ésto son los fusiles que tienen aproximadamente 
el mismo grosor de metal en la boca y en la culata; en cada experiencia hay que pesar la pólvora cuidadosamente y cargarla de manera que no se derrame delante de la bala; sólo se utilizará un taco de estopa en la cantidad necesaria para contener la carga en la cámara; si entre el taco y la bala se deja un espacio vacío habrá que medir su longitud y tenerlo en cuento en los cálculos para la determinación de la velocidad; y la máquina debe de estar suficientemente alejada del cañón del fusil para que la llamarada no pueda alcanzarla e intervenir en el movimiento del péndulo.

Robins se refiere a un péndulo de madera y hierro cuyo peso es de 56 libras y 3 onzas $(2,62 \mathrm{~kg})$, la distancia de su centro de gravedad al eje $E F$ es 52 pulgadas $(1,32 \mathrm{~m})$, la distancia del centro de oscilación al eje $E F 622 / 3$ pulgadas $(1,59 \mathrm{~m})$ y la distancia del centro de la plancha $G H I K$ al eje $E F$ es 66 pulgadas $(1,67 \mathrm{~m})$. Suponiendo que la bala impacta en el centro del bloque, Robins establece la siguiente proporción ${ }^{57}$ :

57 El planteamiento general de un péndulo balistico consiste en reducir el péndulo físico a una masa ideal y puntual situada en el punto de impacto, sobre la cual el efecto sea el mismo, a fin de aplicar las leyes del choque. Sean $m$ la masa de la bala, 0 su velocidad, $M$, la masa del péndulo, $V$ la velocidad del péndulo, e I el momento de inercia. En el caso de un péndulo físico, la longitud reducida o longitud del péndulo simple que le es equivalente es:

$$
d_{0}=\frac{I}{M_{p} d_{8}}
$$

donde esta longitud $d_{o}$ es la distancia del centro de oscilación al eje de giro, y $d_{g}$ lae distancia del centro de gravedad a este mismo eje.

De modo que el momento de inercia:

$$
I=M_{p} d_{0} d_{8}
$$

Por consiguiente, siendo el radio de giro $d_{i}$ la distancia del punto de impacto al eje, el momento cinético que adquiere el péndulo balístico será, llamando $w$ a la velocidad angular de este punto de impacto, y $V$ a su velocidad lineal:

$$
C=I \omega=\frac{v}{d_{i}} d_{0} d_{d} M_{p}
$$

Este momento cinético tiene que ser el mismo que el momento adquirido por la 
cuadrado de la

distancia del centro

del bloque a $\mathrm{EF}$

$(66 \times 66,2,78 \mathrm{~m})$

producto de la distancia

del centro de oscilación

al eje EF por la distancia

de sucentro de gravedad a

$\mathrm{EF}(19,06 \mathrm{~kg})(622 / 3 \times 52 ; 2,09 \mathrm{~m})$

Se sabe que todo cuerpo suspendido de un hilo y puesto en movimiento vibratorio se eleva, describiendo un arco de círculo, hasta la altura que alcanzaría si se le arrojase verticalmente con la velocidad que tiene en el punto más bajo del arco. Esta altura es el seno verso del arco, y la teoría de la caída de cuerpos basta para determinar esta velocidad ${ }^{58}$.

masa puntual ideal $M$ situada en el mismo punto de impacto, que será:

$$
\boldsymbol{C}=\boldsymbol{M V d}
$$

Igualando estas dos últimas ecs. resulta:

$$
\frac{M_{p}}{M}=\frac{d_{i}^{2}}{d_{d} d_{g}}
$$

${ }^{58}$ El problema, en términos actuales, se reduce a aplicar las leyes del choque al encuentro inelástico de la bala, de masa $m$ y velocidad 0 , a la que consideraremos puntual, con la masa puntual $M$. Por la conservación de la cantidad de movimiento, tenemos las ecs.:

$$
m v=(M+m) V
$$

Si ahora aplicamos el principio de conservación de la energía, todavía desconocido en la época, la energía cinética en el momento del choque será igual a la energía potencial debida a la altura $h$ adquirida por el péndulo después del mismo, es decir:

$$
\frac{1}{2}(M+m) V^{2}=(M+m) g h
$$

Si sustituímos el valor de $V$ y simplificamos, tendremos que la velocidad de la bala 
Euler examina los resultados de Robins, siguiendo la misma pauta que en anteriores ocasiones, incluye algunos datos iniciales más y señala dos carencias iniciales en el planteamiento de Robins: 1) hay que considerar el peso de todas las partes del péndulo que intervienen en su movimiento, no sólo el peso de la parte móvil, lo que incluye el travesaño $E F$, y 2) hay que hallar la distancia del centro de gravedad al eje $E F$. La comunicación de la velocidad sigue las leyes del choque. Con los datos proporcionados por Robins, obtiene que la velocidad de la bala del fusil es de $1.583,385$ pies (renanos)/sg, que equivalen a 1.632 pies ingleses $(497,43$ $\mathrm{m} / \mathrm{sg}$ ) y sólo difiere del resultado hallado por Robins, 1.641, en 9 pies/sg (2,74 m/sg). Así pues, Robins no ha cometido ningún error serio de cálculo. Pero sí ha cometido otros. Entre otras cosas, la velocidad real de la bala debe ser mayor de lo que se obtiene en el cálculo, pues hay que tener en cuenta la resistencia que ejerce el aire sobre el movimiento del péndulo. Además, Euler pone de relieve las peculiaridades propias de este tipo de impactos y el porqué no son válidas las reglas habituales para el choque de cuerpos.

Las leyes del choque de cuerpos, elásticos o no, no son suficientes para el caso de dos cuerpos que chocan oblícuamente, es decir, aquéllos cuya impulsión sigue una línea oblícua que no

resulta:

$$
v=\frac{M+m}{m} \sqrt{2 g h}
$$

Ahora bien, siendo $l$ la longitud del péndulo y $\alpha$ el ángulo que éste se desplaza por el impacto:

$$
h=l(1-\cos \alpha)
$$

De donde, siendo sinoer $\alpha=1-\cos \alpha$ (véase fig.3):

$$
\nu=\frac{M+m}{m} \sqrt{2 g l \sin v e r \alpha}
$$


pasa por sus centros de gravedad. El péndulo no se mueve libremente, sino alrededor de un eje, y hay que determinar: 1) el momento de inercia (la suma de los productos obtenidos al multiplicar cada partícula del cuerpo por el cuadrado de su distancia al eje de movimiento), 2) el momento de la fuerza (el producto de la fuerza por la distancia perpendicular de su dirección al eje del movimiento), 3) la fuerza aceleradora absoluta del movimiento alrededor del eje (el momento de la fuerza entre el momento de inercia del péndulo), y 4) el producto de este cociente por la distancia de un punto cualquiera al eje, que dará la fuerza aceleradora de ese punto. Además, es corriente que en la teoría del choque de cuerpos no se preste atención al tiempo necesario para que se produzcan los cambios ocasionados en los dos cuerpos por la percusión, y se habla como si estos cambios fueran instantáneos. Sin embargo, es evidente que la bala necesita un cierto tiempo para hundirse en la plancha de madera, aunque éste sea muy corto. Por tanto, a medida que la bala se hunde en la madera y encuentra la resistencia de ésta, la velocidad del péndulo aumenta y la de la bala disminuye.

Otro factor a tener en cuenta es el punto exacto en que la bala impacta con el péndulo. La regla que da Robins para calcular la velocidad sólo es válida si la bala impacta en el centro de oscilación, si da más arriba la velocidad estimada es demasiado pequeña, y si da más abajo es demasiado grande. Euler, que ya había corregido los resultados experimentales de Robins y la velocidad de $1.641 \mathrm{pies} / \mathrm{sg}$ a $1.632 \mathrm{pies} / \mathrm{sg}$, introduce ahora estos factores y halla que la velocidad de la bala debe ser $1.679 \mathrm{pies} / \mathrm{sg}$ $(511,75 \mathrm{~m} / \mathrm{sg})$. Es decir, que hay una diferencia de $47 \mathrm{pies} / \mathrm{sg}$.

El siguiente paso dado por Robins es la comprobación experimental de las diferencias entre los resultados obtenidos en la práctica con los previstos por la teoría ${ }^{59}$. Para ello realizó 61 disparos en cinco tandas, especificando cuidadosamente las

59 "Proposition X: Déterminer les changemens occasionnés dans la force de la poudre par les différens états de l'atmosphere", ROBINS, opus cit., pp. 176-186. 
características de la pieza, el proyectil y la carga de pólvora. El error de la teoría al calcular la longitud de la cuerda del arco oscila en un margen de cinco pulgadas $(12,7 \mathrm{~cm})$ arriba y abajo. Para los disparos en los que el error era más acusado Robins esgrimía las distintas circunstancias prácticas que forzosamente intervenían en los disparos o errores en el peso de la pólvora, el viento o el impulso de la llama sobre el péndulo. Estaba también el hecho de que en los disparos realizados con cargas muy pequeñas de pólvora el calor no puede alcanzar la misma intensidad que en los hechos con cargas mayores. Por tanto, la fuerza de la pólvora y la elasticidad son menores y esto se deja notar en las experiencias. Véanse las tablas siguientes ${ }^{60}$.

\begin{tabular}{|l|l|l|l|l||}
\hline $\mathrm{N}^{\Omega}$ & $\begin{array}{l}\text { Peso de la } \\
\text { pólvora ("dra- } \\
\text { gmes") }\end{array}$ & $\begin{array}{l}\text { Cuerda del } \\
\text { arco (medida } \\
\text { por la cinta) }\end{array}$ & $\begin{array}{l}\text { Cuerda del } \\
\text { arco (según la } \\
\text { teoría }\end{array}$ & $\begin{array}{l}\text { Error de la } \\
\text { teoría }\end{array}$ \\
\hline 1 & 12 & 18,7 & 19,0 & $+0,3$ \\
\hline 2 & 12 & 19,6 & 19,0 & $-0,6$ \\
\hline 3 & 6 & 13,6 & 13,4 & $-0,2$ \\
\hline
\end{tabular}

Características de las cinco tandas de de disparos:

1) con un cañón (indicado por A)

- diámetro de la bala $=3 / 4$ pulgadas

- longitud del cañón = 45 pulgadas

- longitud de la carga de pólvora = $25 / 8$ pulgadas

- calibre del cañón = 1/40 mayor que el diámetro de la bala

- peso de la carga de pólvora = 12 dracmas ("dragmes"?)

- peso de la bala = 1/12 de libra "avoir du poids"

2) con el mismo cañón

- con una plancha de madera más pesada

- sin que la pólvora llene por completo el espacio de detrás de la bala (AF)

3) un cañón del mismo calibre pero de sólo 12,375 pulgadas de largo (indicado por

C)

- con una plancha de madera algo más ligera

60 ROBINS, opus cit., Prop. IX, pp. 141-145. 


\begin{tabular}{|c|c|c|c|c|c|c|}
\hline $\mathrm{N}^{\mathrm{O}}$ & & $\begin{array}{l}\text { Espacio AF } \\
\text { (detrás de } \\
\text { la bala, en } \\
\text { pulgadas) }\end{array}$ & $\begin{array}{l}\text { Peso de la } \\
\text { pólvora } \\
\text { en dragme }\end{array}$ & $\begin{array}{l}\text { Cuerda del } \\
\text { arco (según } \\
\text { la cinta, en } \\
\text { pulgadas }\end{array}$ & $\begin{array}{l}\text { Cuerda del } \\
\text { arco (según } \\
\text { la teoría, en } \\
\text { pulgadas) }\end{array}$ & $\begin{array}{l}\text { Error } \\
\text { de la } \\
\text { teoría } \\
\text { en pulg. }\end{array}$ \\
\hline 4 & & $25 / 8$ & 6 & 11,9 & 12,1 & 2 \\
\hline 7 & & $11 / 4$ & 6 & 13,9 & 13,6 & -3 \\
\hline 10 & & $25 / 8$ & 12 & 16,9 & 16,8 & -1 \\
\hline 13 & & $25 / 8$ & 6 & 11,1 & 11,5 & +4 \\
\hline 16 & $c$ & $25 / 8$ & 12 & 12,6 & 12,8 & +2 \\
\hline 19 & $A$ & $25 / 8$ & 12 & 17.2 & 17,2 & 0 \\
\hline 22 & A & $25 / 8$ & 6 & 12,4 & 12,2 & -2 \\
\hline 25 & A & $25 / 8$ & 9 & 15,4 & 15,0 & -4 \\
\hline 28 & C & $25 / 8$ & 6 & 8,7 & 9,0 & +3 \\
\hline 31 & $\mathrm{~B}$ & $25 / 8$ & 12 & 14,4 & 14.4 & 0 \\
\hline 34 & A & 4 & 12 & 15,7 & 15,3 & -4 \\
\hline 37 & $A$ & $51 / 4$ & 24 & 11,7 & 11,3 & -4 \\
\hline 40 & A & $13 / 4$ & 8 & 7,6 & 8,1 & +5 \\
\hline 43 & B & $25 / 8$ & 12 & 8,0 & 8,2 & +2 \\
\hline 46 & A & $25 / 8$ & 12 & 9,1 & 9,1 & 0 \\
\hline 49 & $\mathrm{C}$ & $25 / 8$ & 12 & 6,8 & 6,7 & 1 \\
\hline 52 & $\mathrm{C}$ & $25 / 8$ & 6 & 5,0 & 4.8 & 2 \\
\hline 55 & $\mathrm{D}$ & $25 / 8$ & 6 & 4,7 & 4,8 & +1 \\
\hline 58 & $A$ & $21 / 16$ & 6 & 6,4 & 6,5 & +1 \\
\hline 61 & A & $21 / 16$ & 12 & 9,0 & 9,1 & +1 \\
\hline
\end{tabular}


4) con otro cañón de igual calibre que los dos primeros (indicado por B)

n una plancha de madera algo más pesada

5) otro cañón (indicado por D)

- longitud del cañón = 7,09 pulgadas

- calibre del cañón $=0,83$ pulgadas de calibre

- peso de la bala = $331 / 2$ "dragmes"

- con un péndulo mucho más pesado

- peso del péndulo = 97 libras

- distancia del centro de oscilación al eje $=63,9$ pulgadas

Señala Robins que la mayor parte de las experiencias se hicieron y registraron antes de hacer los cálculos teóricos. Reflexionando sobre estos resultados extrae consecuencias tanto para la práctica de la artillería como para la teoría de pólvoras. Su teoría es válida salvo en casos excepcionales, como los que se presentan cuando la carga de pólvora es muy pequeña. Las tablas pueden ser de ayuda para determinar el espesor necesario de una pieza para resistir el esfuerzo de la pólvora y permiten abreviar la preparación del terreno sobre el que se asienta la batería o la pieza.

En cuanto a la discusión sobre la mejor forma con que debe fundirse la cámara del cañón, Robins sostiene, una vez más, que se equivocan quienes creen que se puede sacar un mejor partido de cañones y morteros dependiendo de la forma que se dé a éstas. Insiste ahora en que para obtener con una misma carga la misma velocidad lo que hace falta es que el proyectil esté a la misma distancia del fondo del cañón, pues la misma cantidad de pólvora actúa con más o menos fuerza dependiendo de que el espacio que hay detrás de la bala sea mayor o menor.

Euler se reafirma en algunas de las objeciones planteadas en las anteriores proposiciones (la teoría de Robins sólo es válida si la bala impacta en el centro de oscilación del péndulo) y añade algunas más. Es evidente que Robins ha dejado pasar por alto algunos detalles que, aunque pueden parecer despreciables, cobran importancia si de lo que se trata es de calcular la velocidad del proyectil con la mayor exactitud posible. Por ejemplo, importa, y mucho, determinar el punto de impacto exacto de la bala con el péndulo y su distancia al eje del péndulo. No parece verosímil ni que en todos los disparos las balas hayan impactado siempre a la 
misma altura, ni que se pueda disparar siempre al mismo punto del péndulo, pues entonces las balas chocarían con las que ya se han disparado antes. Puesto que el diámetro de la bala es $3 / 4$ de pulgada $(1,9 \mathrm{~cm})$, los puntos de impacto deben estar alejados entre sí menos de una pulgada. En el caso en que la cuerda $L l$ tenga una longitud de más de 10 pulgadas $(25,4 \mathrm{~cm})$ bastaria con tirar una pulgada más arriba o más abajo para obtener un error de 0,1 pulgadas $(0,25 \mathrm{~cm})$ en su longitud. Una diferencia de 2 pulgadas acarrearía un error de 0,2 pulgadas $(0,5 \mathrm{~cm})$, y una de 3 un error de $0,3(0,7 \mathrm{~cm}), y$ así sucesivamente. Euler sentencia que estos errores proceden de la falta de exactitud en la teoría.

En cuanto a la forma de la cámara, Euler desautoriza a Robins e, indirectamente, reivindica el planteamiento que de este asunto hacía Bélidor. Es fácil ver que la forma de la cámara sí influye en la rapidez con que se inflama la pólvora pues ésta es tanto mayor cuanto más cercanos estén los granos de pólvora entre sí. De todas las formas (esférica, cilíndrica, peroide y cónica) la esférica es aquélla en la que todas sus partes están más próximas y por tanto la inflamación será más rápida. El efecto aun sería mayor si el fuego pudiera iniciarse justo en el centro de la carga, pues desde allí la distancia hacia el resto de la pólvora es menor ${ }^{61}$.

En este asunto Robins navegaba contra corriente. Había un acuerdo generalizado en que la inflamación de pólvora era sucesiva y en que la forma de la cámara afectaba a la rapidez de inflamación de la pólvora. Ni Bélidor ni Euler estaban solos. Precisamente el mismo año en que aparecieron los Nuevos principios ... se realizaron unas experiencias a instancias de la Royal Society con la finalidad de determinar, primero, si toda la pólvora de la carga se inflamaba antes de que la bala se moviese sensiblemente de su lugar, y segundo, si la forma de la cámara, ceteris

61 Bélidor prefería las cámaras conoides. 
paribus, tenía alguna influencia en el alcance del proyectil ${ }^{62}$. El comité designado por la Royal Society desglosó estas dos cuestiones en tres: 1) si se inflama la carga completa, 2) si la bala se empieza a mover antes o después de que se inflame toda la carga, y 3) si la forma de la cámara influía en el alcance.

Se hicieron sucesivos disparos con piezas y cargas de distintas características, algunos de ellos sin bala y sólo con la pólvora. Se colocó una sábana de papel en un marco de madera a cierta distancia para determinar mediante la práctica con exactitud el punto de impacto. En el suelo, entre la pieza y el marco, unas hojas grandes de papel para comprobar con facilidad si quedaban granos de pólvora sin encender que fueran arrojados en el disparo junto con la bala y que cayeran al suelo ${ }^{63}$. Se comprobó lo que los artilleros prácticos sabían ya desde hacía tiempo, que no toda la carga se inflamaba, y se recogieron en cada disparo cierta cantidad de granos de pólvora sin encender, quedando registrados su peso y su composición, cuando esto era factible. Pero a pesar de la exactitud en toda la operación no se extrajo ninguna conclusión ni acerca de la cantidad total de la carga ni de la pólvora que quedaba sin encenderse. Tampoco se extrajo ninguna acerca de la composición. Se observó que junto con la pólvora aparecía polvo, arena y residuos sin determinar. Destaca la existencia de salitre sin mezclar. O bien la pólvora no estaba en perfecto estado de agregación, o bien el azufre y el carbón se consumían por completo pero no así el salitre.

En cuanto a las dos cuestiones restantes, se decide que el proyectil comienza a moverse en el mismo instante en que la carga comienza a inflamarse. Es decir, que la inflamación de la pólvora,

62 "The Report of the Commitee of the Royal Society appointed to examine some Questions in Gunnery", No. 465, p. 172. Read Nov. 4. 1742. Vol VIII Philosophical Transections, 1732-1744. P. 253-259 de la edición citada.

63 La descripción de las concidiciones en que se hicieron los disparos que recogen las Philosophical ... es muy detallada, pero puesto que los pormenores no tienen mayor repercusión en los resultados no me detendré en ella. Pueden consultarse en el lugar ya citado. 
y por tanto la producción de fluido elástico y el desplazamiento de la bala, son sucesivos y no instantáneos. Naturalmente, esto no se pudo demostrar con las experiencias, sino que se adoptó por sentido común y de acuerdo con la comprobación de que no toda la pólvora se inflamaba. Respecto a la forma más ventajosa que se debe dar a la cámara, el comité se inclina, entre varias cámaras todas ellas de igual capacidad, por aquélla de mayor longitud. Es decir, ni la conoide que prefería Bélidor, ni la esférica que prefería Euler. Aquí bien puede emplearse cualquiera de las diferentes advertencias que Euler hace a lo largo de sus comentarios a Robins acerca de la incertidumbre que envuelve a las conclusiones extraídas de experiencias de artillería.

Sorprendentemente, aunque la teoría de Robins es "falsa", hay, en general, una conformidad en los resultados mayor de lo que cabría esperar. No obstante, "Tout cela est trop incertain pour que, de l'accord de l'expérience avec la théorie, on puisse rien conclure en faveur de cette derniere" ${ }^{164}$.

B) Charles Hutton (1775)

Hutton realizaría una nueva determinación de la velocidad de los proyectiles para grandes piezas de artillería en 1775, sirviéndose del diseño unicial del péndulo balístico de Robins.

Charles Hutton, ingeniero militar al igual que Robins y profesor de matemáticas en la Escuela de Artillería de Woolwich ${ }^{65}$, llevó a cabo en 1775 cinco tandas de experiencias con un péndulo balístico, del tipo del de Robins, perfeccionado para ser empleado

64 Ibidem, p. 154.

65 Las primeras noticias sobre la existencia de instalaciones artilleras en Woolwich se retrotaen, al menos, a 1677, fecha en que ya existía en dicho paraje un laboratorio para uso de la artillería. En el XVIII sus pequeñas instalaciones acogían a un centenar de alumnos a cuyo cargo estaba una plantilla de doce profesores (de matemáticas, fortificación, dibujo, química, etc.), en su mayoría ingenieros y oficiales de artillería. 
con balas de cañón ${ }^{66}$. La capacidad matemática de su autor fue reconocida en su tiempo por Montucla, quien dedicó palabras muy elogiosas a sus distintas obras, tanto a los Elements of Conics Sections, como a Miscellaneous Tracts both Physical and Mathematical, y Mathematical Tables...., a la que califica de obra maestra en su género ${ }^{67}$.

La diferencia de su péndulo con el de Robins consistía en que era mayor y más pesado, por lo que podía admitir el impacto de las balas de cañón. En realidad en sus experiencias utilizó dos péndulos, uno de 328 libras de peso (148,78 kg.) y $1021 / 2$ pulgadas avoirdupoids $(2,6 \mathrm{~m}$.), y otro de 552 libras $(250,38 \mathrm{~kg}$.) y 101 pulgadas $(2,56 \mathrm{~m}$.). El cañón empleado era de bronce, con un ánima completamente cilíndrica de 2,16 pulgadas de diámetro en la boca $(5,4 \mathrm{~cm}$.), y 42,6 pulgadas de largo $(1,08 \mathrm{~m}$.). La cámara era cónica, de 2,08 pulgadas de diámetro en el fondo $(5,2 \mathrm{~cm}$.), y el mayor de los proyectiles empleados pesaba $191 / 2$ onzas avoirdupoids $(0,55 \mathrm{~kg}$.). La pólvora era la habitualmente fabricada para el gobierno y las cargas pesaban dos, cuatro y ocho onzas empaquetadas en saquetes (56,69 gr., 113,39 gr. y 226,79 gr.).

$\mathrm{Sean}^{68}: p$ peso de la bala, $P$ peso de todo el péndulo, $d_{8}$ distancia del centro de gravedad del péndulo al eje de movimiento, $d_{o}$ distancia el centro de oscilación al eje de movimiento, $d_{i}$ distancia del centro del punto de impacto (al mismo eje), $v_{2}$ velocidad adquirida por el punto de impacto tras el choque, $v_{1}$, velocidad de

66 HUTTON, Ch.; "The Force of Gun-powder and the initial Velocities of Cannon-balls, determined by Experiments; from which is also deduced the Relation of the initial Velocity to the Wheight of the Shot and the quantity of powder", Communicated by Samuel Horsley, LL.D., Sec. R.S., Philosophical Transactions of the Royal Society of London, vol. LXVIII, for the Year 1778, Part. I, London (1779), pp. 5085.

67 Miscellaneous tracts both Physical and mathematical, Londres (1778). Elements of conics sections, Londres (1787). Esta obra, "dans le goût de la géométrie ancienne" era para Montucla un modelo de precisión y claridad. Véase MONTUCLA; opus cit., Part. V Liv. I, p. 13, pp. 241-242 y 359-360.

68 Aqui he adaptado la demostración de Hutton y modernizado su notación. 
la bala antes del choque, $s$ cuerda del arco medida por la cinta y $r$ radio de este arco o longitud del péndulo

Remitiéndonos a las ecuaciones empleadas anteriormente al hablar de Robins, si introducimos en la ecuación del principio de conservación de la cantidad de movimiento el valor para $M / M_{p}$ dado en la última ecuación de la nota 57, llegamos, con Hutton, a la siguiente expresión:

$$
V=\frac{m d_{i}^{2} v}{m d_{i}^{2}+M d_{g} d_{0}}
$$

que es la misma deducida en casos anteriores. Pero el impacto de la bala ha aumentado la masa del péndulo, alterando la situación. Hutton busca una expresión que corrija este efecto.

Supongamos que ya se ha efectuado un disparo, y la bala se ha incorporado a la masa del péndulo. Ahora, el nuevo valor para la distancia del centro de oscilación al eje de giro será:

$$
d_{0}=\frac{I^{\prime}}{(M+m) d_{8}}
$$

La posición $d^{\prime}{ }_{8}$ del nuevo centro de gravedad resulta:

$$
d g^{\prime}=\frac{M d_{g}+M d_{i}}{M+m}
$$

y el nuevo momento de inercia:

$$
I^{\prime}=m d_{i}^{2}+M d_{0} d_{g}
$$

Sustituyendo estos últimos valores en la ecuación anterior para $d_{0}$ : 


$$
d_{0}^{\prime}=\frac{m d_{i}^{2}+M d_{0} d_{8}}{M d_{i}+M d_{g}}
$$

En este punto, calcula la velocidad $z$ de este nuevo centro de oscilación. Dado que los puntos de impacto y de oscilación tienen la misma velocidad angular, se tiene la proporción:

$$
\frac{V}{d_{i}}=\frac{z}{d_{0}^{\prime}}
$$

Obteniendo, al sustituir el valor de $\mathrm{V}$ dado por la ec.de la pg.anterior, que:

$$
z=\frac{V d_{o}^{\prime}}{d_{i}}=\frac{m d_{i} v}{m d_{i}+M d_{g}}
$$

Por otra parte, el seno verso del arco descrito por el centro de oscilación suministra otra vía para el cálculo de $z$.

En la figura 3 se observa que

$$
x=\operatorname{sinver}(A B) ; y=\operatorname{sinver}(C D)
$$

Haciendo

$$
H=d_{0}^{\prime}
$$

y como

$$
\cos \alpha=\frac{r-x}{r}=\frac{H-y}{H}
$$




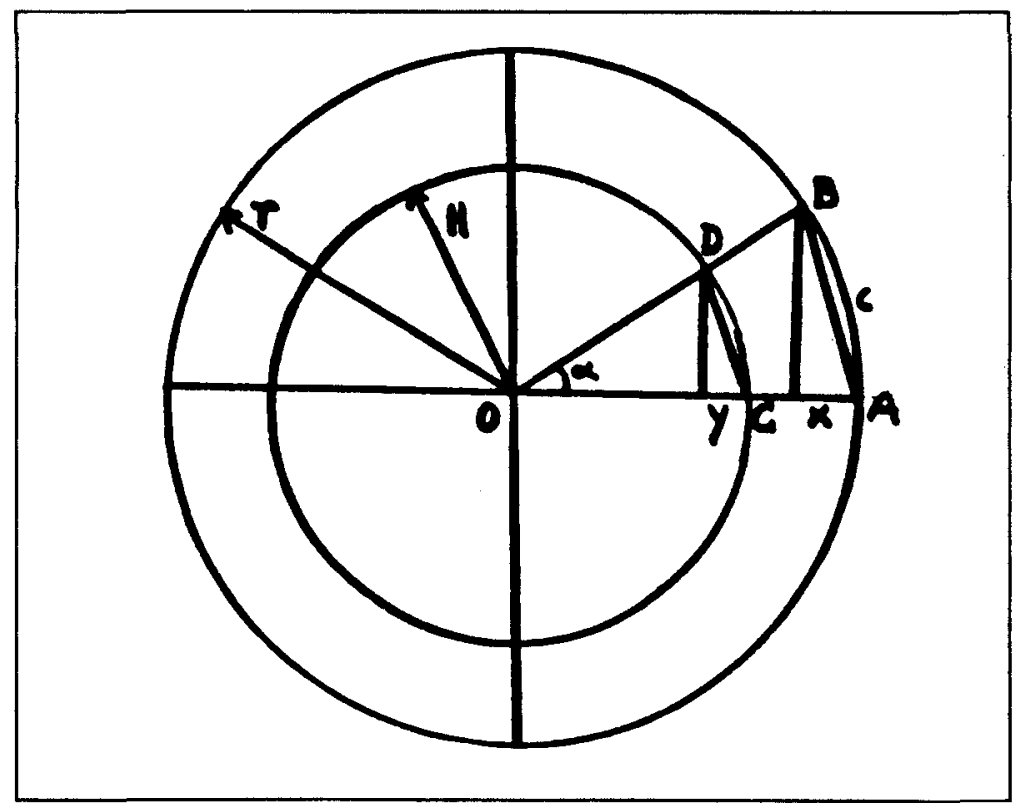

Figura 3

se tiene la proporción:

$$
\frac{r}{H}=\frac{x}{y}
$$

de donde:

$$
y=\frac{x H}{r}=\frac{d_{o}^{\prime}}{r} \operatorname{sinver}(A B)=\frac{d_{o}^{\prime}}{r} \frac{c^{2}}{2 r}=\frac{c^{2} d_{o}^{\prime}}{2 r^{2}}
$$

Pero y representa la altura a la que asciende el centro de oscilación, y la velocidad $z$ adquirida en su descenso será:

$$
z=\sqrt{2 g y}=\sqrt{2 g \frac{c^{2} d_{o}^{\prime}}{2 r^{2}}}=\frac{\sqrt{2 g c}}{r \sqrt{2}} \sqrt{d_{o}^{\prime}}
$$


Véanse, por ejemplo, los resultados obtenidos en la primera y tercera tandas de experiencias realizadas por Hutton ${ }^{69}$.

Primeras series de experiencias realizadas el 13 de Mayo de 1775 , con tiempo claro y seco:

\begin{tabular}{|c|c|c|c|c|c|c|c|c|c|}
\hline $\begin{array}{l}\text { orden } \\
\text { disparos }\end{array}$ & $\left\{\begin{array}{l}\text { carga } \\
\text { onz. }\end{array}\right.$ & $\begin{array}{l}\text { diámet ro } \\
\text { balas } \\
\text { pulgad }\end{array}$ & $\begin{array}{l}\text { longitud } \\
\text { cargas } \\
\text { (pulgad. }\end{array}$ & $k$ & $b$ & $p$ & $g$ & $C$ & $\begin{array}{l}\text { veloc } \\
\text { pies } \\
\text { /sg }\end{array}$ \\
\hline $\begin{array}{l}19 \\
29 \\
39 \\
49 \\
59 \\
69 \\
79 \\
89\end{array}$ & $\begin{array}{l}2 \\
2 \\
2 \\
2 \\
2 \\
2 \\
4 \\
4\end{array}$ & $\begin{array}{l}1,98 \\
1,98 \\
1,98 \\
1,97 \\
1,97 \\
1,96 \\
1,97 \\
1,96\end{array}$ & $\begin{array}{l}3,15 \\
3,15 \\
3,15 \\
3,15 \\
4,5 \\
4,5\end{array}$ & $\begin{array}{l}92,5 \\
92,5 \\
91,6 \\
91 \\
90,5 \\
92,4 \\
92 \\
90,5\end{array}$ & $\begin{array}{l}1,094 \\
1,094 \\
1,094 \\
1,078 \\
1,078 \\
1,063 \\
1,078 \\
1,063\end{array}$ & $\begin{array}{l}323 \\
329,1 \\
330,2 \\
331,5 \\
332,3 \\
333,4 \\
334,4 \\
335,5\end{array}$ & $\begin{array}{l}72 \\
72,1 \\
72,2 \\
72,3 \\
72,3 \\
72,4 \\
72,5 \\
72,5\end{array}$ & $\begin{array}{l}13 \\
17,8 \\
18,1 \\
17,6 \\
16,3 \\
16,2 \\
24 \\
25\end{array}$ & $\begin{array}{l}456 \\
628 \\
647 \\
646 \\
604 \\
598 \\
882 \\
950\end{array}$ \\
\hline
\end{tabular}

Los valores de $k, g$ y $c$ se expresan en pulgadas. Los de $b$ y $p$ en libras.

El péndulo se colocó a 29 de la boca del cañón, la distancia del centro de oscilación $h$ era de 7 pies $1 / 3$ y la longitud total del péndulo $r$ era 102 1/2 pulgadas. Las velocidades de la última columna se calcularon con:

$$
v=5,672 c g \sqrt{h} \frac{p+b}{b k r}
$$

Tercera serie de experiencias realizadas el 12 de Junio de 1775, con tiempo claro, seco y en calma

\begin{tabular}{|c|c|c|c|c|c|c|c|c|c|}
\hline $\begin{array}{l}\text { orden } \\
\text { dis- } \\
\text { paros }\end{array}$ & $\begin{array}{l}\text { arga } \\
\text { onzas }\end{array}$ & $\begin{array}{l}\text { diámet ro } \\
\text { balas } \\
\text { pulg. }\end{array}$ & $\begin{array}{l}\text { Iongitud } \\
\text { cargas } \\
\text { pulg. }\end{array}$ & $k$ & b & $\mathrm{p}$ & $\mathrm{g}$ & & $\begin{array}{l}\text { vel } \\
\text { pies } \\
\text { /sg }\end{array}$ \\
\hline $\begin{array}{l}1 \% \\
29 \\
39 \\
48 \\
59 \\
68\end{array}$ & $\begin{array}{l}2 \\
2 \\
2 \\
4 \\
4 \\
4\end{array}$ & $\begin{array}{l}2,080 \\
2,036 \\
2,045 \\
2,062 \\
2,036 \\
2,045\end{array}$ & $\begin{array}{l}2,85 \\
2,85 \\
2,85 \\
4 \\
4 \\
4\end{array}$ & $\begin{array}{ll}94 & \\
94 & \\
93 & 1 / 2 \\
92 & 1 / 4 \\
93 & 1 / 2 \\
93 & 1 / 2\end{array}$ & $\begin{array}{l}1,219 \\
1,141 \\
1,156 \\
1,188 \\
1,141 \\
1,156\end{array}$ & $\begin{array}{l}324 \\
325,2 \\
326,4 \\
327,5 \\
328,7 \\
329,9\end{array}$ & $\begin{array}{l}71,4 \\
71,5 \\
71,6 \\
71,7 \\
71,7 \\
71,8\end{array}$ & $\begin{array}{l}23 \\
24,5 \\
22 \\
27,3 \\
35 \\
33\end{array}$ & $\begin{array}{r}698 \\
799 \\
715 \\
880 \\
1163 \\
1087\end{array}$ \\
\hline
\end{tabular}

Los valores de $k, g$ y $c$ se expresan en pulgadas. Los de $b$ y $p$ en libras.

Las indicaciones de la tabla anterior son igualmente válidas para ésta.

69 Todas las tandas de experiencias son igualmente ilustrativas e interesantes, por lo que no ha habido un motivo especial para la selección de la primera y la tercera. Tan sólo señalar que en la segunda, realizada el 3 de Junio de 1775 , se notifica que los disparos cuarto y quinto se efectuaron con proyectiles alargados, formados por un cilindro acabado en cada extremo por una semiesfera. ROBINS, opus cit., "Nouvelles expériencies faites à Woolwich pour connoitre les vitesses initiales des boulets", pp. 508-531. 
En general, los resultados obtenidos en todas ellas concuerdan bastante $^{70}$, y en los casos en que se observa alguna discrepancia atribuye la causa a contratiempos de índole estrictamente práctica. En principio podían temerse tres causas de error: 1) los efectos de la penetración de la bala en el péndulo; teóricamente el problema se trata como si la penetración del proyectil fuera instantánea, pero ciertamente no lo es. Ahora bien, se estima que se produce antes de que el péndulo se haya apartado $1 / 10$ de pulgada $(2,54 \mathrm{~mm}$.) de su situación de reposo en $1 / 200$ de segundo, cifras mínimas que se consideran imperceptibles y sin mayor repercusión. 2) la resistencia que el aire opone al movimiento del péndulo; ésta se desprecia puesto que se ejerce contra una velocidad de sólo 3 pies $/ \mathrm{sg}(0,91$ $\mathrm{m}$.), contra una superficie de 20 a 24 pulgadas cuadradas $(0,5$ a 0,6 $\mathrm{m})$ y una masa de no más de 500 libras $(226,8 \mathrm{~kg}$.). 3) el rozamiento de los goznes del péndulo; también es despreciable si se toman las medidas adecuadas, y es contrarrestado por el efecto contrario que produce sobre la distancia al centro de oscilación.

De estas experiencias concluye: 1) la inflamación de la pólvora es casi instantánea; 2) las velocidades comunicadas a balas de igual peso por diferentes cargas de pólvoras son proporcionales aproximadamente a la raíz cuadrada de las cargas; 3 ) las velocidades comunicadas a balas de igual peso por cargas iguales están aproximadamente en proporción inversa a la raíz cuadrada del peso de las balas; 4) las velocidades comunicadas a balas de diferentes pesos por diferentes cargas de pólvora son proporcionales a la raíz cuadrada de las cargas e inversamente proporcionales a la raíz cuadrada de los pesos de las balas; 5 ) sería preferible utilizar balas de forma alargada o bien de una materia más pesada; 6) también sería deseable disminuir el viento de la bala; 7) cumpliendo estos dos últimos puntos podría ahorrarse aproximadamente la mitad de pólvora, pues podría emplearse piezas más cortas y ligeras.

70 Hutton lo califica de "uniformidad asombrosa". 
No satisfecho con los resultados de 1775 Hutton repitió nuevas experiencias con el péndulo en 1783, 1784, $1785 \mathrm{y}$ años sucesivos ${ }^{71}$. Esta vez utilizó cañones de distinto peso y distintas longitudes, y midió la velocidad de las balas por medio del péndulo y del retroceso de las piezas. Ambos procedimientos de determinación arrojan resultados con grandes discrepancias, coincidentes en una única carga, antes y después de la cual los resultados se alejan cada vez más. Esto demuestra que las diferencias ocasionadas en los retrocesos hacen imposible hallar la velocidad real de las balas. Véase la siguiente tabla.

\begin{tabular}{|c|c|c|c|c|c|c|c|c|}
\hline \multirow{3}{*}{$\begin{array}{l}\text { longitud } \\
\text { cańn en } \\
\text { calibres }\end{array}$} & \multicolumn{2}{|c|}{ carga 4 onzas } & \multicolumn{2}{|c|}{ carga 4 onzas } & \multicolumn{2}{|c|}{ carga 8 onzas } & \multicolumn{2}{|c|}{ carga 16 onzas } \\
\hline & \multicolumn{2}{|c|}{ velocidad: } & \multicolumn{2}{|c|}{ velocidad: } & \multicolumn{2}{|c|}{ velocidad: } & \multicolumn{2}{|c|}{ velocidad: } \\
\hline & $\begin{array}{l}\text { retro- } \\
\text { ceso }\end{array}$ & péndulo & $\begin{array}{l}\text { retro- } \\
\text { ceso }\end{array}$ & péndulo & $\begin{array}{l}\text { retro- } \\
\text { ceso }\end{array}$ & $\begin{array}{l}\text { pén- } \\
\text { dulo }\end{array}$ & $\begin{array}{l}\text { retro- } \\
\text { ceso }\end{array}$ & péndulo \\
\hline $\begin{array}{l}15 \\
20 \\
30 \\
40\end{array}$ & $\begin{array}{l}830 \\
863 \\
919 \\
929\end{array}$ & $\begin{array}{l}780 \\
835 \\
920 \\
970\end{array}$ & $\begin{array}{l}1135 \\
1203 \\
1294 \\
1317\end{array}$ & $\begin{array}{l}1100 \\
1180 \\
1300 \\
1370\end{array}$ & $\begin{array}{l}1445 \\
1521 \\
1631 \\
1669\end{array}$ & $\begin{array}{l}1430 \\
1580 \\
1790 \\
1640\end{array}$ & $\begin{array}{l}1345 \\
1485 \\
1680 \\
1730\end{array}$ & $\begin{array}{l}1377 \\
1656 \\
1998 \\
2106\end{array}$ \\
\hline
\end{tabular}

En los disparos hechos con cargas pequeñas la velocidad medida por el retroceso de la pieza es mayor, y a medida que aumentan las cargas las velocidades medidas mediante el péndulo sobrepasan a las medidas por el retroceso, y esto se nota más cuanto más largo es el cañón. Esto se debe a la interacción entre el rozamiento de la plataforma, la velocidad y el retroceso. Los cañones están montados sobre plataformas horizontales, en las que se produce una fricción excesiva de las ruedas con los ejes y de la cola con la cureña. La velocidad, que aumenta con las cargas, hace aumentar

\footnotetext{
71 Véase HUTTON, Charles; "New Experiments in gunnery; for determining the force of fired gunpowder, the initial velocity of Cannon Balls, the Ranges of Projectiles at different Elevations, the Resistance of the Air to Projectiles, the effect of different lenghts of guns and of different quantities of powder", en Tracts on Mathematical and Philosophical Subjects; comprising, among Numerous Important Articles, the Theory of Bridges, with Several Plans of Recent Improvement. Also the Results of Numerous Experiments on the Force of gunpowder, with Applications to the Modern Practice of Artillery. London (1812), 3 vols. Vol. II, Tract XXXIV, PP. 306-384.
} 
también esta fricción. Cuanto más largo es el cañón también es más pesado y una presión de este tipo, aunque sea pequeña, repercute también sobre el retroceso. Por el contrario, en las cargas pequeñas el gas de la recámara encuentra una resistencia mayor a su expansión y se revuelve en el retroceso cuando debería comunicar la velocidad a la bala.

Para tener una visión correcta de las velocidades y alcances conseguidos con una carga determinada es necesario reducir las velocidades medias correspondientes a los últimos intentos a las velocidades iniciales con las que las balas salen de la boca del cañón; debido a la resistencia del aire las que se obtuvieron con una carga de dos onzas tienen que aumentarse en $1 / 36$ y las restantes en $1 / 28$. Véase la siguiente tabla

\begin{tabular}{|c|c|c|c|c|c|c|c|}
\hline $\begin{array}{l}\text { longitud } \\
\text { cañon en } \\
\text { pulgadas }\end{array}$ & $\begin{array}{l}\text { carga } \\
\text { en } \\
\text { onzas }\end{array}$ & $\begin{array}{l}\text { diámetro } \\
\text { bala en } \\
\text { pulgadas }\end{array}$ & $\mid \begin{array}{l}\text { peso bala } \\
\text { en onzas } \\
y \text { dracmas }\end{array}$ & $\begin{array}{l}\text { elevación } \\
\text { cañon en } \\
\text { grados }\end{array}$ & $\begin{array}{l}\text { duracion } \\
\text { vuelo en } \\
\text { segundos }\end{array}$ & alcance & $\begin{array}{l}\text { veloc } \\
\text { bala en } \\
\text { pies/sg }\end{array}$ \\
\hline $\begin{array}{l}38,1 \\
38,1 \\
38,1 \\
38,1 \\
38,1\end{array}$ & $\begin{array}{r}2 \\
2 \\
4 \\
8 \\
12\end{array}$ & $\begin{array}{l}1,96 \\
1,959 \\
1,96 \\
1,962 \\
1.95\end{array}$ & $\begin{array}{ll}16 & 10 \\
16 & 5 \\
16 & 81 / 3 \\
16 & 12 \cdot 1 / 2 \\
16 & 12\end{array}$ & $\begin{array}{l}45 \\
15 \\
25 \\
15 \\
15\end{array}$ & $\begin{array}{r}21,1 \\
9,2 \\
9,2 \\
14,4 \\
15,5\end{array}$ & $\begin{array}{l}5109 \\
4130 \\
4660 \\
6066 \\
6700\end{array}$ & $\begin{array}{r}863 \\
868 \\
1234 \\
1644 \\
1676\end{array}$ \\
\hline 57,4 & 8 & 1,96 & $7 \mathrm{~B}$ & 15 & 10,1 & 5610 & 1938 \\
\hline
\end{tabular}

Como recompensa a sus esfuerzos la Royal Society premió a Hutton con una medalla de oro. Hutton recogió en sus resultados los que ya obtuvo en 1755 y añadió algunos más:

1) balas con igual peso disparadas por cargas distintas adquieren velocidades aproximadamente proporcionales a la raíz cuadrada de la carga;

2) balas de distinto peso disparadas por cargas iguales adquieren velocidades inversamente proporcionales a la raíz cuadrada del peso de la bala;

3) balas de distinto peso disparadas por cargas diferentes adquieren velocidades que son directamente proporcionales a a la raíz cuadrada de la carga e inversamente proporcionales a la del peso de la bala;

4) disminuyendo el viento de la bala se puede llegar a ahorrar hasta $1 / 3$ de la carga, e igualmente si se utilizaran balas de plomo 
o con forma cilíndrica alargada, aunque también esto conlleva algunas desventajas. La bala es el único cuerpo que se encuentra sometido por todas partes y de igual manera a la resistencia (del aire), y aunque se espera de ella que siga una línea de disparo antes o después se aparta de ella. En las experiencias se ha demostrado que la velocidad de la bala y su fuerza de percusión depende del peso específico de su materia. El plomo es el único metal más pesado y más barato que el hierro, pero es demasiado blando y se deforma por la presión de otros cuerpos más duros (por ejemplo, en su recorrido por el interior del ánima del cañón), lo que no lo hace recomendable para el disparo con cañones ni para el tiro de ricochet ${ }^{72}$. Otros metales pesados, como el oro, el platino, la plata o el cobre están fuera de cuestión por su escasez y coste;

5) la velocidad de la bala aumenta con la carga sólo hasta cierto punto, pasado el cual decrece gradualmente. Sin embargo, para conseguir con los grandes cañones una valocidad máxima es necesaria una carga grande, pero ésta no es proporcional a la longitud del cañón, pues hay que tener en cuenta también qué parte de la cámara permanece vacía. Se ha observado un incremente continuo de la velocidad cuanto mayor es la longitud del ánima pero sólo en una proporción muy pequeña. Véase la siguiente tabla.

\begin{tabular}{|l|l|l|l|}
\hline $\begin{array}{l}\text { longitud del } \\
\text { anima en } \\
\text { pulgadas }\end{array}$ & $\begin{array}{l}\text { Iongitud ocupada } \\
\text { por la carga en } \\
\text { pulgadas }\end{array}$ & $\begin{array}{l}\text { fracción ocupada } \\
\text { por la carga } \\
\text { respecto a la } \\
\text { longitud total } \\
\text { delánima }\end{array}$ & $\begin{array}{l}\text { peso de la } \\
\text { carga en } \\
\text { onzas }\end{array}$ \\
\hline 28,2 & 8,2 & $3 / 10$ & 12 \\
38,1 & 9,5 & $3 / 12$ & 14 \\
57,4 & 10,7 & $3 / 16$ & 16 \\
79,3 & 12,1 & 20 & 18 \\
\hline
\end{tabular}

72 El disparo de ricochet consiste en hacer que el proyectil rebote contra el suelo una o más veces antes de impactar sobre el objetivo. 
6) los alcances aumentan con las cargas en una proporción menor que las velocidades, aproximadamente como la raíz cuadrada. $\mathrm{Si}$ compara con la proporción de las velocidades y la longitud de la pieza se observa que con un aumento considerable de esta última sólo se consigue un pequeño aumento en el alcance que es aproximadamente proporcional a la raíz de la quinta potencia de la longitud del ánima. Con una longitud doble del cañón sólo se consigue 1/7 más de alcance; 7) la duración del vuelo del proyectil es proporcional al alcance; 8) sobre la velocidad del proyectil no influyen ni los distintos pesos de los cañones, ni la posición del oído o fogón ni los respectivos ángulos de elevación; 9) las desviaciones accidentales del proyectil del plano vertical pueden llegar a ser, con un ángulo de $15^{\circ}$, de hasta $1 / 4$ del alcance.

Como se ve, partiendo de la resolución de un problema en apariencia estrictamente matemático, como es la determinación de la velocidad de los proyectiles, se establecieron abundantes consecuencias de interés tanto para la fabricación de pólvoras como para la fundición de piezas de artillería, y se produce el encuentro entre la teoría matemática y la práctica artillera. 